\title{
CYCLOPOIDA SIPHONOSTOMA (CRUSTACEA) VON BANYULS (FRANKREICH, PYRÉNÉES.ORIENTALES) MIT BESONDERER BERUUCKSICHTIGUNG DES GAST-WIRTVERHÄLTNISSES
}

von

KARL SCHIRL

Institut für taxonomische Zoologie (Zoölogisch Museum), Universität von Amsterdam, Niederlande ${ }^{1}$ )

\section{SUMMARY}

The sponges, Clathrina clathrus, $\mathrm{Cl}$. lacunosa, $\mathrm{Cl}$. primordialis, and $\mathrm{Cl}$. contorta, as well as Oscarella lobularis, have been checked on the presence of associated siphonostome cyclopoids. Two new species have been found, Asterocheres simplex n. sp. and A. corneliae n. sp. Moreover, the hitherto unknown males of Asterocheres complexus and $A$. mucronipes have been described. Six species have been found on new hosts, for three species the hitherto unknown host has been discovered (Rhynchomyzon falco on Clathrina contorta, Acontiophorus antennatus on $\mathrm{Cl}$. lacunosa and on Oscarella lobularis, Myzopontius pungens on $\mathrm{Cl}$. primordialis and on $\mathrm{Cl}$. contorta). Furthermore, evidence is provided that certain species of siphonostomes are predominantly found on certain hosts. So, it can be said that Asterocheres simplex frequents Clathrina clathrus and $\mathrm{Cl}$. lacunosa, that $\mathrm{Myzo}$ pontius pungens frequents Clathrina contorta, and that Asterocheres mucronipes frequents Oscarella lobularis. Asterocheres corneliae is most abundant on Clathrina primordialis, but on the same host one finds also numerous Myzopontius pungens.

Finally, a list of all known hosts for siphonostomes is provided, based on an extensive (though certainly not complete) search in the literature.

\section{EINLEITUNG}

Dank eines Stipendiums des Ministeriums für Unterricht und Wissenschaft der Niederlande war es mir möglich, diese Untersuchung vorzunehmen. Professor J. H. Stock stellte mir dazu sein gesammeltes Material zur Verfügung, wofür ich ihm - sowie für seine grosse Unterstützung bei der Arbeit - aufrichtig danke. Ebenfalls will ich noch Prof. J. Vacelet aus Marseille für die Bestimmung verschiedener Wirttiere (Schwämme) danken.

In vielen Veröffentlichungen über die parasitische Copepodengruppe der Asterocheriden steht

1) Jetzige Adresse: I. Zool. Inst. Univ. Wien, A 1190, Wien, Oesterreich. leider nur allzuoft hinter dem Wort "Wirt" ein Fragezeichen. Soweit ich aus der mir zur Verfügung stehenden Literatur entnehmen konnte, beträgt die gesamte Artenzahl der Asterocheriden nun über 170. Dem steht gegenüber, dass nur von 68 Arten der Wirt bekannt ist. Angaben wie "zwischen Algen" und "auf Schwämmen" sind dabei nicht mitgezählt. Vor allem den älteren Autoren war eine genaue Wirtsangabe unmöglich, wie wir aus Giesbrecht's Werk "Die Asterocheriden des Golfes von Neapel" auf Seite 199, 2. Absatz entnehmen können: "Wenn man aber fragt, aus welchen Tieren oder Pflanzen die Asterocheriden ihre Nahrung saugen, so ist es eben ihre rasche Fähigkeit des Ortswechsels, die eine Beantwortung dieser Frage durch die direkte Beobachtung von vornherein unwahrscheinlich macht. Denn es leuchtet ein, dass Tiere, die sich beunruhigen, leicht durch die Flucht entziehen können, dies auch bei den Belästigungen tun werden, denen sie während des Dredgens ihrer Wirte ausgesetzt werden müssen. Wenn man das Dredgenmaterial durchsucht, wird man kaum erwarten können, die Asterocheriden noch an ihren geschüttelten, gepressten, umhergeworfenen Wirten vorzufinden. Man müsste die als Wirte verdächtigen Tiere einzeln und behutsam dem Meere entnehmen, um ihre Asterocheriden feststellen zu können."

Prof. J. H. Stock versuchte nun schon längere Zeit gezielt Wirte zu sammeln. Die Vorgangsweise bei dem hier vorgelegenen Material - allein Schwämmen - war folgende: mittels Tauchgeräte wurden die Schwämme gesammelt und an Ort und Stelle in Plastiksäckchen gegeben. Das Material wurde dann in getrennten Schalen ausgesucht und in Röhrchen mit 70 prozentigem Alkohol konserviert. So konnte man einer Vermengung der Tiere zuvorkommen. 
Doch nicht allein die Zuweisung eines Wirtes war das Ziel dieser Arbeit. Es drängten sich weitere Fragen auf: sind die Asterocheriden omnivor, oder kann man eine Bevorzugung eines Wirtes feststellen? Wie ist die Artenkombination auf einem Wirt? Besteht eine Beziehung zwischen Wirt und der Morphologie des Parasiten? - Diese und ähnliche Fragen stellten sich bei dieser Arbeit, welche teilweise aufgehellt, jedoch noch nicht endgültig beantwortet werden können.

Siebzehn Proben von 5 verschiedenen Schwämmen wurden in dieser Arbeit untersucht. Vier davon sind Kalkschwämme (Clathrina-Arten), einer ein Kieselschwamm (Oscarella lobularis). Die ursprüngliche Nummerierung, Datum, Fundort und Tiefe seien hier vermerkt:
Asterocheres complexus Stock, 1960; Ummerkutty, 1966.

Gesammeltes Material. -

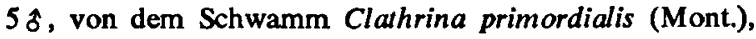
Cap Oullestreil, $\pm 10 \mathrm{~m}, 26.8 .1962$.

Diese Art wurde 1960 von Stock, ebenfalls in Banyuls, auf dem Schwamm Spongelia fragilis (Schmidt) gefunden. In Clathrina primordialis liegt nun ein neuer Wirt vor. Stock sammelte nur Weibchen. Darum will ich hier eine Beschreibung der sexuellen Unterschiede beifügen. Diese liegen vor im Urosom, in der ersten Antenne, den Maxillipeden und am zweiten Ruderfuss.

Das Urosom des Männchens (Fig. 5a) besitzt drei freie Glieder zwischen Furca und Genitalsegment. Davon ist das Analsegment mehr als

\begin{tabular}{|c|c|c|c|c|}
\hline Schwamm & Nummerierung & Fundort & Tiefe & Datum \\
\hline $\begin{array}{l}\text { Clathrina } \\
\text { clathrus }\end{array}$ & $\left\{\begin{array}{l}61-61 \\
61-65 \\
62-8 \\
62-12 \\
63-15\end{array}\right.$ & $\begin{array}{l}\text { Cap Réderis } \\
\text { Pier Banyuls } \\
\text { Pier Banyuls } \\
\text { Cap Réderis } \\
\text { Cap Béar }\end{array}$ & $\begin{array}{l}26 \mathrm{~m} \\
\pm 10 \mathrm{~m} \\
\pm 10 \mathrm{~m} \\
20-24 \mathrm{~m} \\
22 \mathrm{~m}\end{array}$ & $\begin{array}{r}25.8 .1961 \\
2.9 .1961 \\
2.8 .1962 \\
17.8 .1962 \\
23.7 .1963\end{array}$ \\
\hline $\begin{array}{l}\text { Clathrina } \\
\text { lacunosa }\end{array}$ & $\left\{\begin{array}{l}61-52 \\
61-71 \\
62-3\end{array}\right.$ & $\begin{array}{l}\text { Cap de l'Abeille } \\
\text { Pier Banyuls } \\
\text { Cap Réderis }\end{array}$ & $\begin{array}{l}20 \mathrm{~m} \\
\pm 10 \mathrm{~m} \\
22-24 \mathrm{~m}\end{array}$ & $\begin{array}{r}28.8 .1961 \\
8.9 .1961 \\
9.8 .1962\end{array}$ \\
\hline $\begin{array}{l}\text { Clathrina } \\
\text { primordialis }\end{array}$ & $\left\{\begin{array}{l}61-70 \\
62-9 \\
62-36 \\
63-17 \\
63-22 \\
63-23\end{array}\right.$ & $\begin{array}{l}\text { Pier Banyuls } \\
\text { Pier Banyuls } \\
\text { Cap Oullestreil } \\
\text { Pier Banyuls } \\
\text { Cap de l'Abeille } \\
\text { Cap de l'Abeille }\end{array}$ & $\begin{array}{l} \pm 10 \mathrm{~m} \\
\pm 10 \mathrm{~m} \\
\pm 10 \mathrm{~m} \\
10-12 \mathrm{~m} \\
10-17 \mathrm{~m} \\
\pm 15 \mathrm{~m}\end{array}$ & $\begin{array}{r}8.9 .1961 \\
2.8 .1962 \\
26.8 .1962 \\
27.7 .1963 \\
30.7 .1963 \\
30.7 .1963\end{array}$ \\
\hline $\begin{array}{l}\text { Clathrina } \\
\text { contorta }\end{array}$ & $65-49$ & Les Trois Moines & $10-14 \mathrm{~m}$ & 2.8.1965 \\
\hline $\begin{array}{l}\text { Oscarella } \\
\text { lobularis }\end{array}$ & $\left\{\begin{array}{l}61-49 \\
62-11\end{array}\right.$ & $\begin{array}{l}\text { Cap Réderis } \\
\text { Cap Réderis }\end{array}$ & $\begin{array}{l}26 \mathrm{~m} \\
20-24 \mathrm{~m}\end{array}$ & $\begin{array}{l}25.8 .1961 \\
17.8 .1962\end{array}$ \\
\hline
\end{tabular}

Das ganze Material stammt aus dem Mittelmeer und wurde in der Nähe von Banyuls (Pyrénées-Orientales) gesammelt. Die Lage der Fundorte ist aus der Karte (Fig.7) ersichtlich.

\section{SYSTEMATISCHER TEIL}

Zwölf Arten von siphonostomen Cyclopoiden wurden gefunden. Zwei davon sind neu für die Wissenschaft. Die Genera Asterocheres, Rhynchomyzon und Acontiophorus gehören zur Familie der Asterocheridae, die restlichen Genera Myzopontius und Pteropontius zu den Artotrogidae.

\section{Asterocheres complexus Stock, 1960}

Asterocheres boecki; Giesbrecht, 1899, non Brady; Ascomyzon latum Sars, 1915 (part.). doppelt so lang wie die zwei anderen Segmente. Merkwürdigerweise waren die medianen Cilienreihen, die beim Weibchen dorsal liegen, auf allen Urosomgliedern unauffindbar. Anscheinend sind diese beim Männchen sehr reduziert und nur auf den lateralen Zipfeln des Genitalsegmentes als kurze Querreihen sichtbar. (Pfeil bei Fig. 5a).

Die erste Antenne (Fig. 5b) ist 18gliedrig. Die Segmente sechzehn und siebzehn sind mehr als doppelt so lang wie breit. Die Bewehrung der einzelnen Segmente ist wie folgt: I:1; II:1; III:1; IV:1; V:1; VI:1; VII:1; VIII:1; IX:3; X:1; XI:I; XII:2; XIII:1+A; XIV:1; XV:1; XVI:1; XVII:2; XVIII: $6+A$.

Die Maxillipeden (Fig. 2h) tragen einen auffallend spitzen, triangulären Fortsatz am Beginn 
des zweiten Handgliedes. Die Endklaue ist etwas länger als das zweite Handglied.

Weiters ist ein sexueller Dimorphismus am Endopoditen des zweiten Ruderfusses (Fig. 5c) vorhanden. Das dritte Glied besitzt distal einen flammenförmigen Fortsatz, darunter eine breite Einsenkung mit einem kurzen Haar. Ebenso sind die distalen Haare kürzer als gewöhnlich.

Verbreitung: Golf von Neapel (Giesbrecht), Banyuls (Stock, Schirl), Golf von Manner, India (Ummerkutty).

\section{Asterocheres mucronipes Stock, 1960}

A. mucronipes Stock, 1960 : 224-228; 1966a : 146-147.

Gesammeltes Material. -

29, vom Schwamm Clathrina clathrus (Schmidt), Pier Banyuls, $\pm 10 \mathrm{~m}$. 2.8.1962.

$2 \%, 4 \hat{\delta}$, derselbe Wirt, Cap Réderis, 20-24 m. 17.8 . 1962.

5 ㅇ, 2 ô, auf dem Schwamm Clathrina lacunosa (Johnston), Cap de l'Abeille, 20 m. 28.8.1961.

$1 \%$, auf dem Schwamm Clathrina primordialis (Mont.), Cap Oullestreil, \pm 10 m. 26.8.1962.

19, derselbe Wirt, Cap de l'Abeille, $10-17$ m. 30.7. 1963.

$40 \%, 56 \hat{\delta}$, auf dem Schwamm Oscarella lobularis (Schmidt), Cap Réderis, 20-24 m, 17.8.1962.

25 ㅇ, 20 \& , derselbe Wirt, Cap Réderis, $26 \mathrm{~m}, 25.8 .1961$.

Stock fand diese Art 1960 auch in Banyuls auf der Gorgonie Eunicella stricta und in Mauritius, 1966, auf Oscarella sp. Die Grössenangaben der von Stock beschriebenen Weibchen liegen zwischen 0,55 und $0,66 \mathrm{~mm}$. Die nun gefundenen $q$ \% messen 0,62 bis $0,76 \mathrm{~mm}$; ein Exemplar sogar 0,90 mm. Die Tiere sind also etwas grösser wie bei Stock. Sie sind auch auffallend breit. So misst ein Tier von $0,70 \mathrm{~mm}$ Länge $0,58 \mathrm{~mm}$ in der Breite. Die Sipholänge gibt Stock mit "bis zu Pl reichend" an. Bei den meisten der mir vorliegenden Exemplare reicht der Sipho über den Ansatz von P2 hinaus, bei einigen - vor allem bei den Männchen - sogar über P3. Männliche Vertreter wurden nun zum ersten Mal gefunden.

Die Grössenangaben der Männchen liegen zwischen $0,58-0,64 \mathrm{~mm}$. Der Mittelwert beträgt $0,60 \mathrm{~mm}$. Die Breite beträgt bei einem Tier von $0,58 \mathrm{~mm}$ Länge $0,41 \mathrm{~mm}$, bei $0,60 \mathrm{~mm}$ Länge 0,42 $\mathrm{mm}$. Sexuelle Unterschiede sind im Urosom, in den ersten Antennen, in den Maxillipeden und in den dritten Ruderfüssen zu finden.

Das Urosom (Fig. 5d) wirkt gedrungen. Das Genitalsegment erweckt den Anschein, als ob es das letzte Metasomasegment sprengen würde.
Die erste Antenne (Fig. 5e) besteht aus 17 Gliedern. Davon sind die Glieder fünfzehn und sechzehn länger als die anderen. Die Bewehrung sieht folgendermassen aus: I:1; II:2; III:2; IV:1; V:2; VI:1; VII:2; VIII:2; IX:3; X:I; XI:1; XII:1; XIII:2; XIV:2; XV:1; XVI:2+A; XVII:4.

Die Maxillipeden sind kräftig gebaut und besitzen am zweiten Basalglied einen stumpfen, triangulären Fortsatz. Die Endklaue wirkt kurz und plump.

P3 (Fig. 5f): das dritte Glied der Endopoditen ist auffallend lang und schmal (die Länge ist viermal so gross wie die Breite) und besitzt an der Aussenseite eine charakteristische Eindellung, worin ein sehr kurzes Haar sitzt. Bei einem Exemplar fand ich auf dem dritten Glied des Exopoditen an der Aussenseite statt drei Dornen nur zwei. Nach Überprüfung mehrerer Exemplare musste ich dieses Merkmal als individuelle Abweichung dieses einen Exemplares betrachten. Die Ausbildung des dritten Ruderfusses beim Männchen stimmt genau mit der von Asterocheres stimulans (cf. Giesbrecht, 1899, Taf. 3 Abb. 1-8) überein.

Verbreitung: Banyuls (Stock, Schirl), Mauritius (Stock).

\section{Asterocheres parvus Giesbrecht, 1897}

Asterocheres parvus Giesbrecht, 1897, 1899; Canu, 1898; Stock, 1960.

Ascomyzon echinicola Sars, 1915; Brady, 1923; Lang, 1949.

Gesammeltes Material. -

1 ô, auf dem Schwamm Clathrina lacunosa (Johnston), Cap Réderis, 22-24 m. 9.8.1962.

1 o auf dem Schwamm Clathrina primordialis (Mont.), Cap Oullestreil, $\pm 10 \mathrm{~m}$. 26.8.1962.

Lang (1949) fand Asterocheres parvus auf Halichondria sp., Stock (1960) auf Spongelia fragilis. Clathrina lacunosa und Clathrina primordialis sind demgemäss neue Wirte für diesen parasitären Cyclopoiden. Doch handelt es sich dabei sicher nicht um typische Wirte. Das dürfte eher Halichondria sein, denn Lang spricht von "vielen 9 ㅇ und $\sigma^{*} \sigma^{*}, "$ die er darauf gefunden hat.

Verbreitung: Neapel (Giesbrecht), Normandie (Ca$\mathrm{nu}$ ), Norwegen (Sars), Sardinien (Brian), Gasörännan (Lang), Banyuls (Stock, Schirl).

\section{Asterocheres latus (Sars, 1915)}

Ascomyzon latum Sars, 1915.

Gesammeltes Material. -

1\%, auf dem Schwamm Clathrina primordialis, Cap Oullestreil, $\pm 10 \mathrm{~m}$. 26.8.1962. 
Sars fand Asterocheres latus auf einer Alge, Lang auf einer Rotalge. Clathrina primordialis ist ein neuer Wirt.

Verbreitung: Norwegen (Sars), Smedjan (Lang), Banyuls (Schirl).

\section{Asterocheres genodon Stock, 1966a}

A. genodon Stock, 1966a; Stock, 1966b.

Gesammeltes Material. -

4 , auf Clathrina primordialis, Cap Oullestreil, $\pm 10 \mathrm{~m}$. 26.8.1962.

Bisher bekannte Wirte waren: Haliclona sp. (Stock 1966a, Mauritius) und Siphonochalina communis (Stock 1966b, Golf von Aqaba). Clathrina primordialis ist wieder ein neuer Wirt für Asterocheres genodon.

Verbreitung: Mauritius (Stock), Golf von Aqaba (Stock), Banyuls (Schirl).

\section{Asterocheres simplex n. sp.}

Gesammeltes Material. -

34 , vom Schwamm Clathrina clathrus (O. Schmidt), Pier Banyuls, $\pm 10 \mathrm{~m}$. 2.9.1961.

4 \% , derselbe Wirt, Cap Réderis, 26 m. 25.8.1961.

$30 \%$, derselbe Wirt, Pier Banyuls, \pm 10 m. 2.8.1962.

33 \%, derselbe Wirt, Cap Réderis, 22-24 m. 17.8.1962.

$20 \%, 4 \delta$, derselbe Wirt, Cap Béar, 22 m. 23.7.1963.

$5 \%$, vom Schwamm Clathrina lacunosa (Johnston), Cap

Réderis, 22-24 m. 9.8.1962.

3 \%, derselbe Wirt, Cap de l'Abeille, 20 m. 28.8.1961.

$5 \%$, derselbe Wirt, Pier Banyuls, $\pm 10 \mathrm{~m}$. 8.9.1961.

$7 \%$, vom Schwamm Clathrina primordialis (Mont.),

Pier Banyuls, $\pm 10 \mathrm{~m}$. 8.9.1961.

8 \%, derselbe Wirt, Pier Banyuls, \pm 10 m. 2.8.1962.

$3 \%$, derselbe Wirt, Cap Oullestreil, $\pm 10 \mathrm{~m}$. 26.8.1962.

$9 \%$, derselbe Wirt, Pier Banyuls, 10-12 m. 27.7.1963.

$19 \%, 1 \delta$, derselbe Wirt, Cap de l'Abeille, $10-17 \mathrm{~m}$. 30.7.1963.

$1 \%$, derselbe Wirt, Cap de l'Abeille, $\pm 15 \mathrm{~m}$. 30.7.1963.

1 , vom Schwamm Oscarella lobularis (O. Schmidt), Cap Réderis, $20-24$ m. 17.8.1962.

3 \%, derselbe Wirt, Cap Réderis, 26 m. 25.8.1961.

Aus einer Probe von Clathrina clathrus (Cap Béar, 22 m; 23.7.1963) wurde ein Weibchen zum Holotypus, ein Männchen zum Allotypus erklärt. Alle anderen Exemplare sind Paratypen (ZMA. Co. 102.367 a-c).

Beschreibung. -

Weibchen: Totale Länge, die Furcaläste ausgeschlossen, 0,52-0,58 mm (Mittelwert: 0,55 mm, gemessen an zehn Exemplaren).

Die Körperform ist normal cyclopoid (Fig. 1a), Das erste fusstragende Segment ist dem Cephalothorax einverleibt. Die folgenden drei Segmente verschmälern sich leicht. Das vierte, freie Metasomasegment setzt sich deutlich gestielt vom dritten Segment ab und dient als Ansatzglied für das fünfte Fusspaar.

Das Urosom (Fig. 1b) baut sich aus vier Segmenten auf. Das erste Abdominalsegment ist mit dem vierten Metasomasegment verschmolzen. Das Genitalsegment (Fig. 1b) ist merklich breiter als lang. Die Verhältniszahlen sind b:1 $=1,7: 1$.

Die Segmentmitte ist am breitesten. Hinter der Geschlechtsöffnung befindet sich ein lateraler Ciliensaum, dessen Cilienlänge schnell $\mathrm{zu}$ - und wieder abnimmt. Die Eiersäcke enthalten je fünf grosse, mit perforatem Muster versehene Eier. Das dritte Urosomsegment ist rund doppelt so breit wie lang. Das Analsegment ist kaum merklich breiter als länger; die Breite verhält sich zur Länge wie 1,3 zu 1.

Die Furca wieder ist - was die Aussenseite betrifft - kaum nennenswert länger als breit. Die Innenseite ist um ein Viertel kürzer als die Aussenseite. Doch ist die Furca um die Hälfte kürzer als das Analsegment. Die Furcaläste sind folgendermassen angeordnet: an der Aussenseite ein dünnes Haar; anschliessend ein kräftiger, gefiederter Ast mit zwei dünnen, dorsal querliegenden Haaren; dann folgt der stärkste und längste, ebenfalls gefiederte Ast; an der Innenseite befindet sich noch ein dünnes, relativ kurzes Haar.

Die erste Antenne (Fig. 1c) ist 20gliedrig; die Bewehrung ist wie folgt: I:1; II:1; III:1; IV:1; $\mathrm{V}: 1$; VI:1; VII:0, sehr kurz; VIII:2; IX:0; X:3; XI:I; XII:1; XIII:2; XIV:1; XV:1; XVI:1; XVII: 1; XVIII:1+A; XIX:2; XX:9, davon eines sehr lang. Der Endteil des zwanzigsten Gliedes ist verschmälert und mittels einer schrägen Querlinie etwas abgesetzt, was auf eine Verschmelzung zweier Glieder schliessen lässt. Die ersten 10 Segmente sind ungefähr um ein Drittel breiter als die folgenden. Die gesamte Länge der ersten Antenne erreicht nicht den Hinterrand des Cephalothorax.

Die zweite Antenne (Fig. 1d) hat einen aus zwei Segmenten bestehenden Protopodit. Das zweite Segment davon ist lang und trägt den Exopodit. Dieser ist vier Mal so lang wie breit und reicht beinahe bis zur Hälfte des ersten Endopoditengliedes. Terminal befinden sich zwei ungleich lange Haare, wovon das längere das erste Endopoditensegment überragt. Der Endopodit besteht aus drei Segmenten. Das erste ist beinahe so lang wie das zweite Protopoditenglied; das zweite ist dreieckig, zwischen dem ersten und dritten 


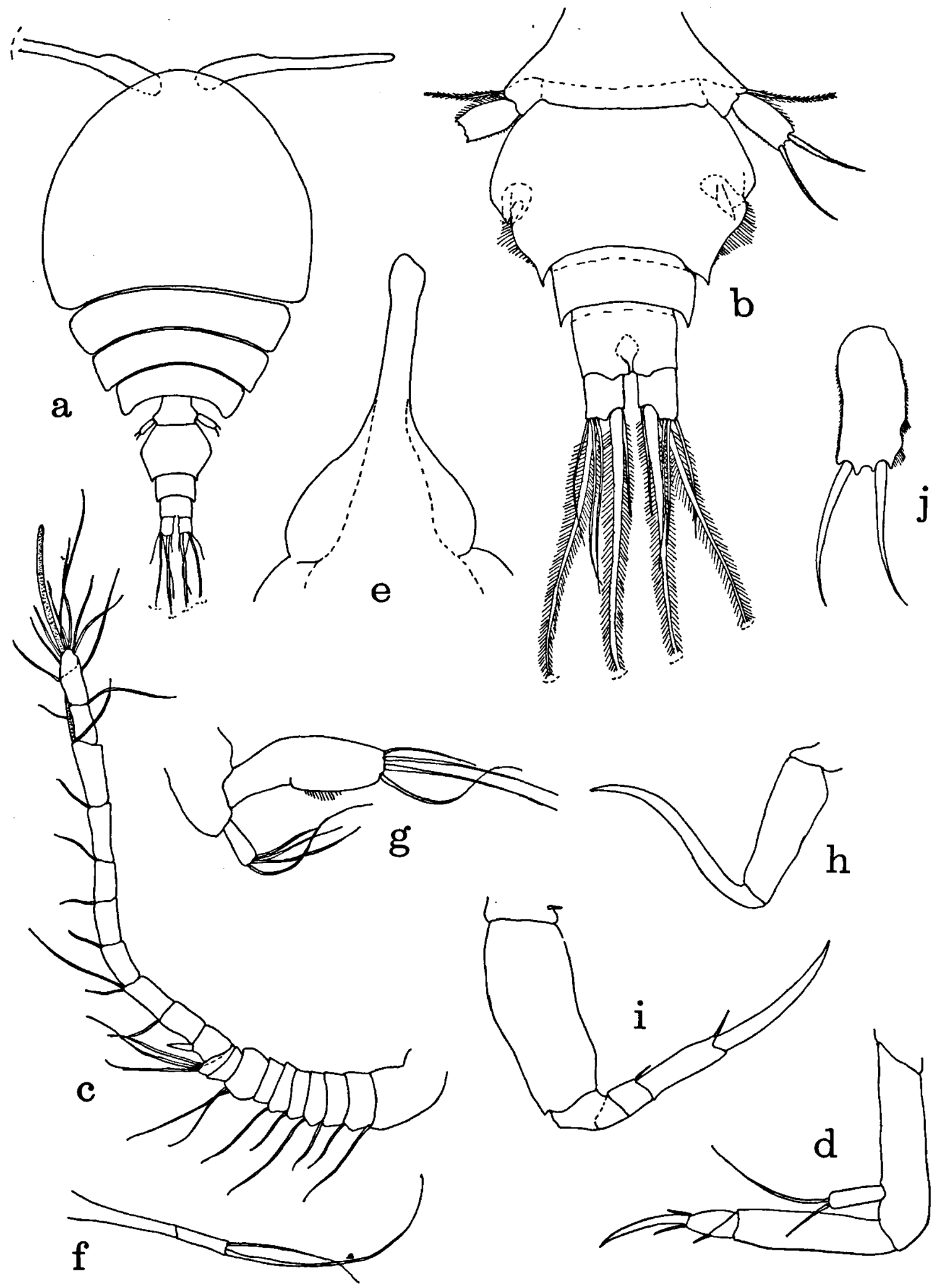

Fig. 1. Asterocheres simplex n. sp., Weibchen: a, ganzes Tier, dorsal (Massstab I); b, Urosom, ventral (II); c, erste Antenne (II); d, zweite Antenne (III); e, Sipho (III); f, Mandibelpalpus (III); g, erste Maxille (III); h, zweite Maxille (III); i, Maxillipede (III); $\mathfrak{j}$, fünfter Fuss (IV). 
Glied eingeklemmt und trägt ein kurzes Haar; das dritte besitzt ein subterminales Haar und zwei terminale Elemente: eine kurze Endklaue (Länge ca. die Hälfte des ersten Endopoditengliedes) und ein Haar, dessen Länge ein Drittel der Endklaue beträgt.

Der Sipho (Fig. 1e) ist wohl tubiform, doch sehr kurz. Seine Länge beträgt durchschnittlich $0,10 \mathrm{~mm}$ und er erreicht kaum die Ansatzstelle der Maxillipeden.

Der Mandibelpalpus (Fig. 1f) ist zweigliedrig; das erste Glied ist zwei Mal so lang wie das zweite. Terminal sitzen zwei ungleich lange Haare.

Die 1. Maxille (Fig. 1g): der Endopodit besitzt an der Aussenseite eine charakteristische Knickkerbe; auf derselben Seite befindet sich noch ein Ciliensaum. Der Exopodit ist an der Basis schmal und verbreitet sich nach oben hin konisch; die Länge davon beträgt zirka ein Viertel des Endopoditen. Sowohl der Exo- als auch der Endopodit tragt 4 terminale Haare.

Die 2. Maxille (Fig. 1h) besteht aus einem relativ schmalen Basisglied und einer schmalen, etwas längeren Endklaue (Verhältniszahl Basis: Klaue $=1: 1,5$ ).

Der Maxilliped (Fig. 1i) trägt am ersten Glied ein kurzes Haar. Die Klaue ist aus drei Segmenten aufgebaut. Segment 3 und 4 tragen je ein Haar. Die Endklaue ist so lang wie die Segmente 2, 3 und 4. Das terminale Haar von Segment 4 ist kurz (Verhältniszahl Haar: Klaue $=1: 6$ ).

Die Ruderfüsse 1 bis 4 (Fig. 2a, b, c, d) besitzen Endo- und Exopoditen, die jeweils 3gliedrig sind. Intercoxalplatten sind vorhanden. Die Basipoditen sind mit lateralen Haaren versehen. Die Coxopoden tragen je ein medianes Haar, das beim zweiten und dritten Fuss gefiedert ist. Das zweite Segment aller Endopoditen ist an der distalen Aussenseite zu nur einer Spitze ausgezogen, was ein Charakteristikum dieser Art ist. Alle Asterocheres-Arten, ausser A. indicus Sewell, 1949, besitzen hier 2 Spitzen. Am dritten Endopoditenglied sind stets 2 terminale Fortsätze zu finden, wovon der äussere länger ist. Doch ist der innere Fortsatz stets vorhanden.

Besprechung der einzelnen Ruderfüsse:

1. Ruderfuss (Fig. 2a): Am ersten Segment des Exopoditen befindet sich ein mächtiger Dorn an der Aussenseite, ein kurzes, dünnes, ungefiedertes Haar an der Innenseite. Das dritte Glied des Endopoditen ist auffallend breit und gedrungen; die Länge beträgt zirka 1,5 Mal die Breite.
2. Ruderfuss (Fig. 2b): Normal asterocherid.

3. Ruderfuss (Fig. 2c): Normal asterocherid.

4. Ruderfuss (Fig. 2d): Das erste Endopoditenglied trägt an der Innenseite ein kurzes, dünnes, ungefiedertes Haar.

Die Chätotaxisformel für P1-P4 lautet:

$$
\begin{aligned}
& \text { P } 1\left\{\begin{array}{lrr}
\text { exp. } I-(1) ! & (\mathrm{I})-1 & \text { (III) }-4 \\
\text { enp. } 0-1 & 0-2 & 1-5
\end{array}\right. \\
& \text { P } 2 \quad\left\{\begin{array}{llc}
\text { exp. I-1 } & \text { I }-1 & \text { III }-\mathrm{I}-4 \\
\text { enp. } 0-1 & 0-2 & \text { (1) }-5
\end{array}\right. \\
& \text { P } 3\left\{\begin{array}{lll}
\text { exp. I-1 } & \text { I }-1 & \text { III }-\mathrm{I}-4 \\
\text { enp. } 0-1 & 0-2 & \text { (2) }-\mathrm{I}-3
\end{array}\right. \\
& \text { P } 4 \quad\left\{\begin{array}{llc}
\text { exp. } I-(1) ! & \mathrm{I}-1 & \mathrm{III}-\mathrm{I}-4 \\
\text { enp. } 0-1 & 0-2 & 2-\mathrm{I}-(2)
\end{array}\right.
\end{aligned}
$$

Die Klammern besagen, dass diese Haare oder Dornen etwas kleiner als gewöhnlich sind. Die mit Klammern und Rufzeichen versehenen Angaben sind sehr reduziert und auch nicht gefiedert. Dornen sind mit römischen Ziffern geschrieben, Haare mit arabischen.

Das fünfte Fusspaar (Fig. 1j) ist reduziert. Der Gliedstummel ist rund zwei Mal so lang wie breit; er erreicht die Genitalöffnung nicht. Die Oberseite erscheint gewellt und ist mit einem Ciliensaum versehen. Die Unterseite ist mit sehr kurzen Härchen ausgestattet. Terminal befinden sich zwei kahle, ungefähr gleich lange Haare. Das gefiederte Basishaar überragt den Gliedstummel.

Die Farbe der Tiere ist nicht ganz einheitlich: die Exemplare, die von Clathrina clathrus stammen, besitzen einen schwach durchscheinenden, etwas gelblichen Körper und gelbliche Eier. Die auf Clathrina primordialis gesammelten Individuen hingegen sind - sowohl was den Körper als auch die Eier betrifft - schwach durchscheinend weisslich. Doch kann man bei den Eiern einen Ubergang van weisslich nach orange, je nach dem Reifungszustand, feststellen. Offensichtlich können sich die Tiere an den Wirt in ihrer Färbung anpassen (Clathrina clathrus ist gelblich, Clathrina primordialis weiss).

Männchen: Totale Länge, die Furcaläste wieder ausgeschlossen: 0,47 mm. Das Urosom (Fig. 2e) besteht aus fünf Segmenten. Das Genitalsegment ist breiter als lang (die Breite verhält sich zur Länge wie 1,4 zu 1) und an den äusseren Unterseiten verlängert, woran zwei Haare sitzen (Reste des sechsten Ruderfusspaares). Die Abdominalsegmente 3 und 4 sind breit und kurz. Das Analsegment besitzt die Länge von Segment 3 und 4 zusammen. 

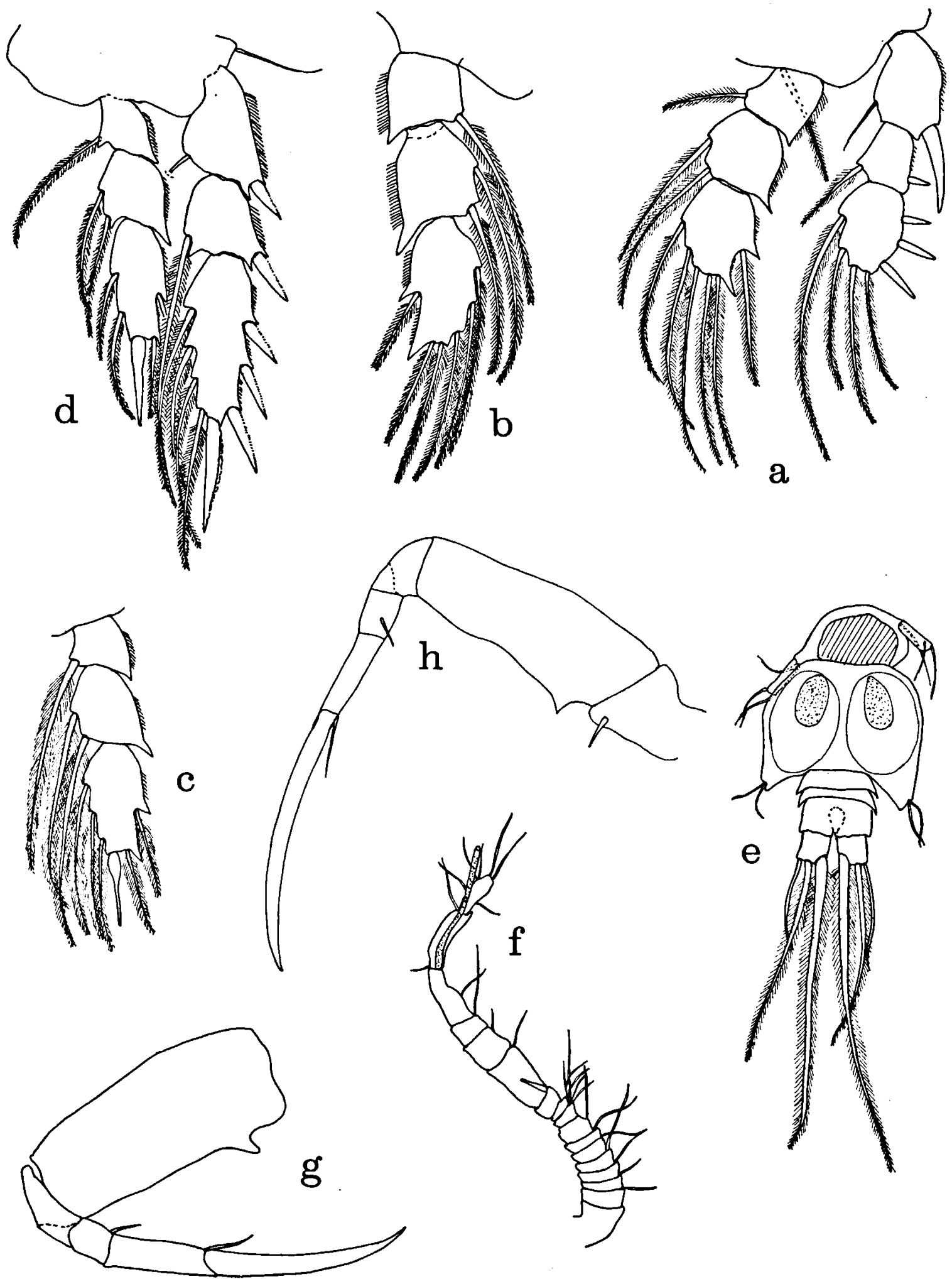

Fig. 2. Asterocheres simplex n. sp., Weibchen (a-d): a, erster Ruderfuss (Massstab III); b, zweiter Ruderfuss, Innenast (III); c, dritter Ruderfuss, Innenast (III); d, vierter Ruderfuss (III). Asterocheres simplex n. sp., Männ-

chen (o-g): e, Urosom (II); f, erste Antenne (II); g, Maxillipede (III). Asterocheres complexus Stock, 1960, Männchen (h): h, Maxillipede (III). 
Andere sexuelle Unterschiede sind an der ersten Antenne und den Maxillipeden zu finden.

Die erste Antenne (Fig. 2f) ist 17gliedrig. Die ersten elf Segmente stimmen mit denen des Weibchens überein. Segment 11 trägt ebenfalls einen Dorn. Die Glieder 12, 15 und 16 sind merklich länger als die anderen Glieder. Segment 15 trägt den Sinneskolben; Segment 16 besitzt terminal einen Fortsatz.

Die Maxillipeden (Fig. 2g) haben im ersten Drittel des zweiten Basalgliedes einen Fortsatz, der zum ersten Basalglied hin gebogen ist.

Zusammenfassung der charakteristischen Merkmale dieser Art:

2. Antenne: langer Exopodit (ungefähr die Hälfte des Endopoditengliedes 1).

1. Maxille: Kerbe an der Aussenseite des Endopoditen.

P1-P4: 2. Glied des Endopoditen mit nur einer distalen Spitze.

P1: kurzes, ungefiedertes Haar an der Innenseite des ersten Exopoditengliedes.

P4: kurzes, ungefiedertes Haar an der Innenseite des ersten Exopoditengliedes.

Auf Grund der Sipholänge (Ansatz der Maxillipeden) stehen folgende Arten Asterocheres simplex nahe: Asterocheres abyssi Hansen, 1923 (1), A. alter Eiselt, 1965 (2), A. bacescui (Marcus, 1965) (3), A. canui Giesbrecht, 1897 (4), A. halichondriae Stock, 1966a (5), A. indicus Sewell, 1949 (6), A. jeanyeatmanae Yeatman, 1970 (7), A. orientalis Sewell, 1949 (8), A. scutatus Stock, 1966b (9), A. uncinatus Kričagin, 1873 (10).

Art (1) ist $\mathrm{zu}$ gross $(0,88 \mathrm{~mm})$ und hat ein $\mathrm{zu}$ langes Analsegment.

Art (2) besitzt lange, konvergierende Furcasegmente; der Exopodit der 2. Antenne ist zu kurz und P5 hat drei terminale Haare.

Art (3) hat die Furca zu lang, die erste Antenne besteht aus 21 Segmenten, der Mandibelpalpus ist eingliedrig.

Bei Art (4) sind Weibchen unbekannt; die erste Antenne des Männchens baut sich aus 18 Gliedern auf, die proximale Borste des Exopoditen der ersten Maxille ist verdickt.

Bei Art (5) besteht die erste Antenne aus 19 Gliedern, an P2 finden wir beim Männchen einen sexuellen Dimorphismus vor, P5 besitzt drei Haare.

Art (6) ist $\mathrm{zu}$ gross $(0,71 \mathrm{~mm})$, hat keine laterale Cilienreihe am Genitalsegment und die erste Antenne besitzt 19 Gliedern.

Art (7) ist $\mathrm{zu}$ gross $(0,68-0,79 \mathrm{~mm})$, hat $21 \mathrm{Seg}$ mente in der ersten Antenne und eine andere Anzahl der Eier (7).

Art (8) Grösse: 0,88 mm, drei Haare an P5.

Art (9) 19 Segmente an der ersten Antenne, einteiliger Mandibelpalpus, drei Haare an P5.

Art (10) Grösse: 0,85-0,90 mm, Cilienquerreihen am Abdomen, erste Antenne mit 21 Gliedern, der Exopodit der zweiten Antenne besitzt nur ein terminales Haar.

Alle hier genannten Arten - ausser Asterocheres indicus Sewell, 1949 - besitzen am zweiten Endopoditenglied zwei distale Spitzen, und alle - ausser Asterocheres halichondriae Stock, 1966a in P4 - haben nicht die reduzierten Haare an der Innenseite des 1. Exopoditengliedes von P1 und P4.

Der Name "simplex" bezeichnet, dass nur eine Zacke am zweiten Glied des Endopoditen vorhanden ist.

\section{Asterocheres corneliae n. sp.}

Gesammeltes Materiai -

7 , auf dem Schwamm Clathrina clathrus (Schmidt), Pier Banyuls, $\pm 10 \mathrm{~m}$. 2.9.1961.

2\%, derselbe Wirt, Cap Réderis, $26 \mathrm{~m}$. 25.8.1961.

8 , derselbe Wirt, Pier Banyuls, $\pm 10 \mathrm{~m}$. 2.8.1962.

2 \%, derselbe Wirt, Cap Réderis, 20-24 m. 17.8.1962.

$7 \%, 3 \hat{\circ}$, auf dem Schwamm Clathrina primordialis (Mont.), Pier Banyuls, \pm 10 m. 8.9.1961.

$10 \%$, derselbe Wirt Pier Banyuls $\pm 10 \mathrm{~m}$. 2.8.1962.

182 으, derselbe Wirt, Cap Oullestreil, $\pm 10 \mathrm{~m}$. 26.8 . 1962.

11 \%, derselbe I. irt, Cap de l'A beille, 10-17 m. 30.7. 1963.

2 \%, derselbe Wirt, Cap de l'Abeille, $\pm 15 \mathrm{~m} .30 .7 .1963$.

$7 \%$, auf dem Schwamm Clathrina contorta (Bowerbank), Les Trois Moines, 10-14 m. 2.8.1965.

Aus einer Probe von Clathrina primordialis (Pier Banyuls, $\pm 10 \mathrm{~m} ; 8.9 .1961$ ) wurde ein Weibchen zum Holotypus, ein Männchen zum Allotypus erklärt. Alle anderen Exemplare sind Paratypen (ZMA. Co. 102.368 a-c).

\section{Beschreibung. -}

Weibchen: Totale Länge, die Furcaläste ausgeschlossen, 0,52-0,70 mm (Mittelwert, gemessen an 15 Exemplaren, 0,56 mm). Die Körperform ist länglich oval (Fig. 3a). Das erste fusstragende Segment ist dem Cephalosoma einverleibt; daher kann man von einem Cephalothorax sprechen. $\mathrm{Er}$ ist viermal breiter als lang. Die Metasomasegmente 1 und 2 sind kaum merklich schmäler als der Cephalothorax, das dritte Segment um ein Drit- 


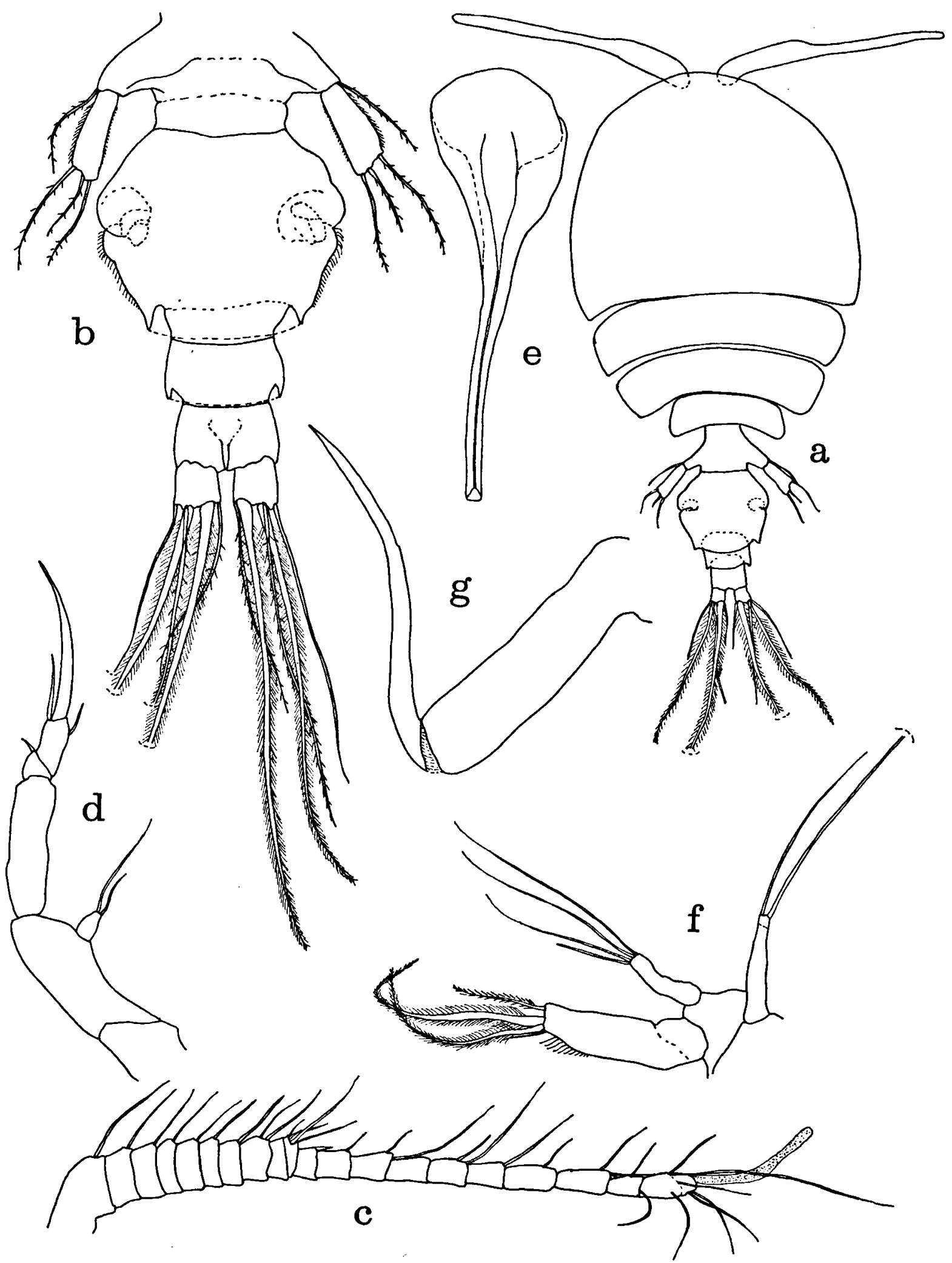

Fig. 3. Asterocheres corneliae n. sp., Weibchen: a, ganzes Tier, dorsal (Massstab I); b, Urosom, ventral (II); c, erste Antenne (II); d, zweite Antenne (III); e, Sipho (III); f, erste Maxille und Mandibelpalpus (III); g, zweite Maxille (III). 
tel. Das vierte Metasomasegment setzt sich deutlich gestielt vom dritten Segment ab.

Das Urosom (Fig. 3b) besteht aus vier Segmenten. Das erste Urosomsegment ist mit dem vierten Metasomasegment verschmolzen und trägt das fünfte, rudimentäre Fusspaar. Das Genitalsegment besitzt seine grösste Breite etwas über der Genitalöffnung (die Länge verhält sich zur Breite wie $7 \mathrm{zu}$ 8,3). Die Genitalöffnungen liegen in der Segmentmitte und sind mit einer komplizierten Chitinstruktur versehen; ein Chitinzahn davon stellt den Rest des sechsten Fusspaares dar. Unter der Genitalöffnung befindet sich eine halbkreisförmige Ausbuchtung; hier beginnt ein lateraler Saum von kurzen Cilien, der sich bis zum Ende des Segments fortsetzt. Die distalen Ecken sind etwas rectangulär; dieser Eindruck wird noch durch die durchscheinende Chitinstruktur verstärkt. Das dritte Urosomsegment ist deutlich schmäler als das Genitalsegment und gleich lang wie das Analsegment. Dieses ist breiter als lang (die Länge verhält sich zur Breite wie 2,1 zu 3,4). Die Furca ist um ein Drittel kürzer als das Analsegment und ungefähr quadratisch. Die Anordnung der Furcaläste sieht folgendermassen aus: aussen ein dünnes, kahles Haar; anschliessend ein kräftiger, dicht gefiederter Ast mit einem dorsal querliegenden, dünn gefiedertem Haar; darauf folgt der stärkste und längste, ebenfalls dicht gefiederte Ast. Das Innenhaar ist das kürzeste, dünn und leicht gefiedert.

Die erste Antenne (Fig. 3c) ist 20gliedrig. Bewehrung: I:1; II:1; III:1; IV:1; V:1; VI:1; VII: 1; VIII:2; IX:0; X:4; XI:I; XII:1; XIII:2; XIV:0; XV:2; XVI:1; XVII:1; XVIII:1+A; $\mathrm{XIX}: 2 ; \mathrm{XX}: 6$. Die ersten zehn Segmente sind breiter und kürzer als die folgenden. Segment $\mathbf{X X}$ ist aus der Verschmelzung zweier Segmente entstanden. Die gesamte Länge der ersten Antenne reicht ungefähr bis zum Ende des Cephalothorax.

Die zweite Antenne (Fig. 3d) besitzt einen aus zwei Segmenten bestehenden Protopoditen. Das zweite Segment davon ist gegen das Ende hin etwas ausgebuchtet, worauf der Exopodit sitzt. Dieser ist eingliedrig, kurz (ein Fünftel des ersten Endopoditengliedes), etwas flaschenförmig und trägt zwei terminale, gleich lange Haare. Der Endopodit besteht aus drei Segmenten. Das erste ist schlank und etwas kürzer als das zweite Protopoditenglied. Das zweite Segment ist dreieckig, trägt ein kurzes Haar und ist zwischen den benachbarten Segmenten eingeschoben. Das dritte Segment ist länglich. besitzt ein subterminales Haar und zwei terminale Elemente: die Endklaue, die lang und gebogen ist (ihre Länge entspricht den Endopoditsegmenten 1,2 und 3), und ein Haar, das fast die Hälfte der Endklaue erreicht.

Der Sipho (Fig. 3e) ist deutlich tubiform, schlank, und überragt etwas die Basis des ersten Ruderfusspaares.

Der Mandibelpalp (Fig. 3f) verjüngt sich im letzten Drittel flaschenförmig; er ist wohl zweigliedrig, doch ist die Trennlinie kaum sichtbar. Das Verhältnis zwischen basalem und distalem Teil lautet 8:1. Terminal sitzen zwei gleich lange kahle Haare.

Erste Maxille (Fig. 3f): der Endopodit ist viermal so lang wie breit, besitzt an der Innenseite einen Ciliensaum und trägt terminal vier ungleich lange Haare. Drei sind gefiedert; zwei davon sind länger als der Endopodit, das dritte ist halb so lang wie die zwei anderen; das vierte ist kahl und sehr kurz (zirka ein Viertel der zwei langen Haare). Der Exopodit erreicht die Hälfte des Endopoditen; er trägt terminal vier kahle Haare. Drei davon sind lang und überragen den Endopoditen beträchtlich, das vierte erreicht kaum dessen Rand.

Die zweite Maxille (Fig. 3g) ist schlank; die Endklaue ist ungefähr um ein Viertel länger als das Basisglied und besitzt an der Unterseite etwas über der Mitte eine kleine Einkerbung.

Die Maxillipeden (Fig. 4e) haben eine zweigliedrige Basis; das erste Glied davon trägt ein kurzes Haar. Die Klaue besteht aus drei Segmenten. Das erste ist aus zwei Segmenten verschmolzen. Eine angedeutete Trennlinie und ein Dorn an der Grenze geben das an. Das zweite Segment ist kurz und mit einem kurzen Haar versehen. Das dritte Glied ist doppelt so lang wie das zweite, trägt ein Haar und die Endklaue, dessen Länge mit den Gliedern $1+2+3$ übereinstimmt. Das Längenverhältnis des Endhaares vom dritten Glied und der Endklaue lautet 1:5. An der Gelenkstelle zwischen dem zweiten Basisglied und der Klaue befindet sich eine abgegrenzte Chitinplatte. Einzigartig ist der Dorn in der Mitte der Oberseite des ersten Klauensegmentes.

Die Ruderfüsse 1 bis 4 (Fig. 4a, b, c, d) besitzen alle dreigliedrige Endo- und Exopoditen. Intercoxalplatten sind vorhanden, die Basipoditen mit lateralen Haaren versehen. Die Coxopoditen tragen je ein medianes Haar. Die distale Aussenseite des zweiten Gliedes aller Endopoditen ist mit zwei Zacken versehen. Am dritten Endopoditenglied von P2 bis P4 sind zwei terminale Fortsätze 


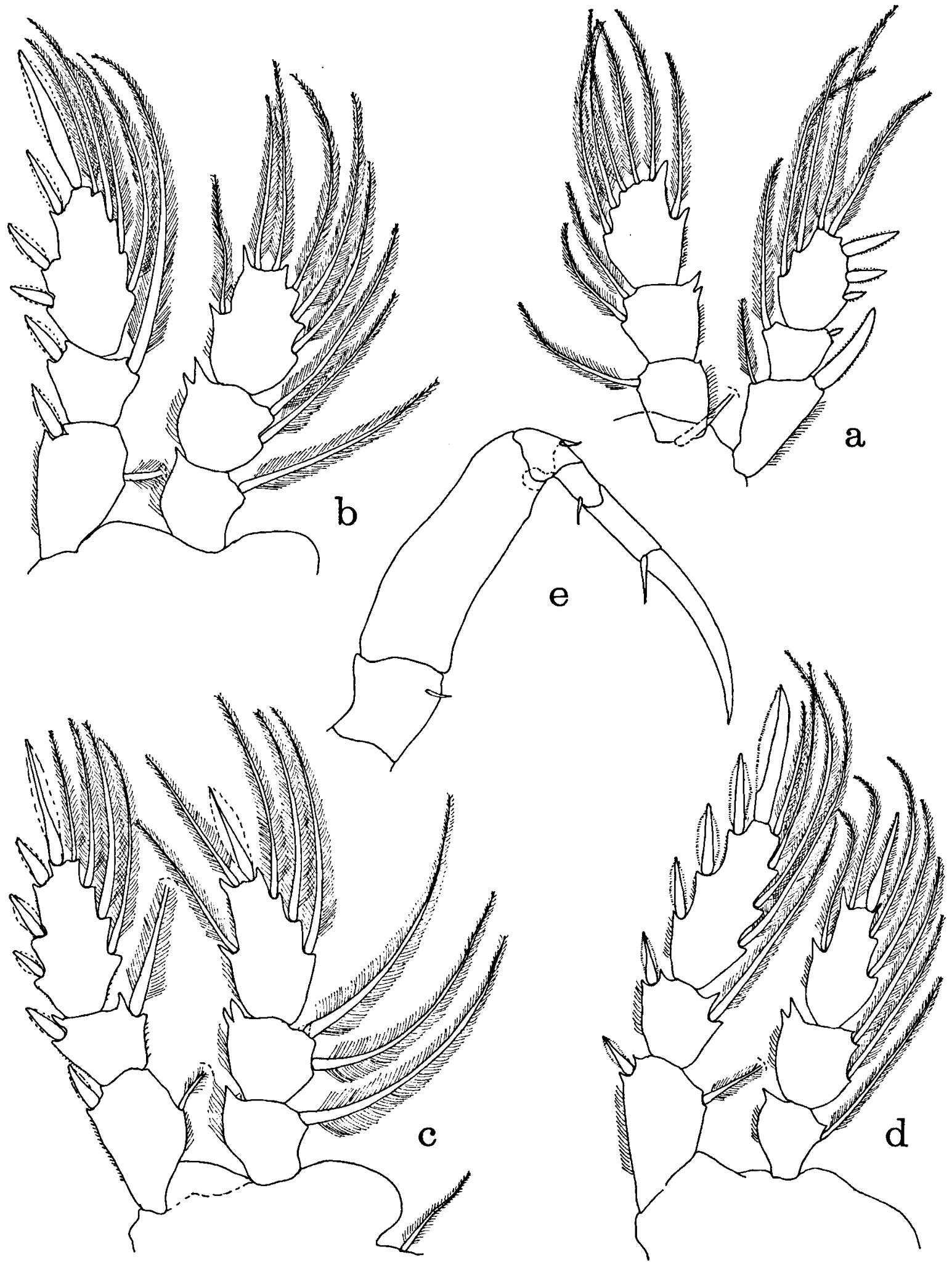

Fig. 4. Asterocheres corneliae n. sp., Weibchen: a, erster dritter Ruderfuss (III); d, vierter Ruderfuss (III); e, Ruderfuss (Massstab III); b, zweiter Ruderfuss (III); c, Maxillipede (III). 
zu finden, am ersten Fuss allein an der Aussenseite. Alle Exopoditen besitzen ein langgestrecktes erstes Segment. An der Aussenseite des ersten Segmentes des ersten Ruderfusses sitzt ein sehr grosser Dorn, der beinahe die Hälfte des dritten Gliedes erreicht; an der Innenseite befindet sich ein kurzes, doch normal gefiedertes Haar (Fig. 4a). P2 (Fig. 4b) und P4 (Fig. 4d) tragen die volle Anzahl Haare und Dornen, ohne grössere Abweichungen. Der Exopodit des dritten Ruderfusspaares (Fig. 4c) trägt am ersten Segment an der Innenseite ein kurzes, schwaches Haar. Die Chätotaxisformel lautet:

\begin{tabular}{|c|c|c|c|}
\hline P 1 & $\begin{array}{l}\text { exp. } I-(1) \\
\text { enp. } 0-1\end{array}$ & $\begin{array}{l}\text { (I) }-1 \\
0-2\end{array}$ & $\begin{array}{r}\text { (I)II }-4 \\
1-5\end{array}$ \\
\hline $\begin{array}{l}P 2 \\
\end{array}$ & $\begin{array}{l}\text { exp. } I-1 \\
\text { enp. } 0-1\end{array}$ & $\begin{array}{l}I-1 \\
0-2\end{array}$ & $\begin{array}{c}\mathrm{III}-\mathrm{I}-4 \\
1-5\end{array}$ \\
\hline P 3 & $\begin{array}{l}\text { exp. } I-(1) \\
\text { enp. } 0-1\end{array}$ & $\begin{array}{l}I-1 \\
0-2\end{array}$ & $\begin{array}{r}\mathrm{III}-\mathrm{I}-4 \\
2-\mathrm{I}-3\end{array}$ \\
\hline P 4 & $\left\{\begin{array}{l}\text { exp. } I-1 \\
\text { enp. } 0-1\end{array}\right.$ & $\begin{array}{l}I-1 \\
0-2\end{array}$ & $\begin{array}{r}\mathrm{III}-\mathrm{I}-4 \\
2-\mathrm{I}-2\end{array}$ \\
\hline
\end{tabular}

Die Klammern besagen, dass diese Haare oder Dornen etwas reduziert sind.

Das fünte Fusspaar (Fig. 3b) ist reduziert; der Gliedstummel ist ungefähr dreimal so lang wie breit, erreicht aber die Genitalöffnung nicht. Sowohl die laterale als auch die mediane Seite ist mit einem Ciliensaum versehen. Terminal sitzen drei Haare. Das mittlere ist kürzer als die zwei anderen. Von oben gezählt sind die zwei ersten breitgestreut gefiedert, das dritte nur in der Endregion. Das Basishaar ist ebenfalls leicht gefiedert und überragt etwas den Gliedstummel.

Farbe der Tiere: durchscheinend weiss.

Männchen: Totale Länge der Tiere, die Furcaläste ausgeschlossen, $0,41 \mathrm{~mm}$. Das Urosom (Fig. $5 \mathrm{~g}$ ) besteht aus fünf Segmenten; das Genitalsegment ist breiter als lang (die Länge verhält sich zur Breite wie $3 \mathrm{zu}$ 5). Die folgenden drei Segmente sind ungefähr gleich lang, doch ist jedes davon etwas schmäler als das Vorhergehende. Die Furca ist mit der des Weibchens identisch. Weitere sexuelle Unterschiede finden wir in der ersten Antenne, der zweiten Maxille, dem Sipho und in den Ruderfüssen P3 und P5.

Die erste Antenne (Fig. 5h) ist 18gliedrig. Die ersten zehn Glieder sind sehr breit und kurz, doch mit denen des Weibchens identisch. Glied 11 trägt ebenfalls einen Dorn, Glied 12 ist auffallend länger als die benachbarten Glieder. Das siebzehnte Segment trägt einen Sinneskolben.

Der Sipho ist etwas länger als beim Weibchen; er überragt die Basis des zweiten Ruderfusses.

Die zweite Maxille (Fig. 5i) ist kräftiger gebaut, die Endklaue kürzer und dicker als beim Weibchen.

Die Maxillipeden besitzen keinen Kopulationshaken am zweiten Basalglied.

Der Processus spiniformis des Endgliedes von P3 ist kürzer als der des Weibchens (Fig. 5j). Auch sind alle Endglieder von P1 bis P4 kräftiger und breiter gebaut als beim Weibchen. Doch ist die Chätotaxisformel dieselbe. Der Gliedstummel von P5 ist, mit dem des Weibchens verglichen, schlanker. Die Länge ist fünfmal so gross wie die Breite.

Obwohl diese Art keine in die Augen springende Kennzeichen hat, muss sie doch als neue Art bezeichnet werden, da sie in den Details keiner der bisher bekannten Arten entspricht. Folgende Besonderheiten seien zusammenfassend aufgezählt: der auffallend lange Stiel zwischen Metasomasegment 3 und 4; die halbkreisförmigen Ausbuchtungen hinter den Genitalöffnungen; das characteristische Teilverhältnis der Mandibelpalpglieder; die Kürze des Innenrandhaares des Endopoditen der ersten Maxille, sowie deren Länge des Exopoditen, namentlich die Hälfte vom Endopoditen (diese letztgenannten Kennzeichen finden wir nur bei wenigen Arten: $A$. jeanyeatmanae, $A$. major, $A$. ovalis und $A$. scutatus); der Dorn am ersten Klauenglied der 2. Maxillipeden; reduzierte Haare am ersten Exopoditenglied von P1 und P3; die weitgestreute, dünne Befiederung der Haare an P5; nur ein dorsales Haar bei den Furcaanhängen.

Auf Grund der Sipholänge stehen folgende Arten nahe: Asterocheres boecki Sars, 1915 (1); A. complexus Stock, 1960 (2); A. ellisi Hamond, 1968 (3); A. genodon Stock, 1966a (4); A. intermedium (Hansen, 1923) (5); A. latus (Sars, 1915) (6); A. micheli Gurney, 1927 (7); A. mucronipes Stock, 1960 (8); A. parvus Giesbrecht, 1897 (9); $A$. tenerum (Hansen, 1923) (10). Doch liegen folgende Unterschiede vor:

Art (1) Grösse: † $0,90 \mathrm{~mm}$; Teilungsverhältnis des Mandibelpalps (1:4); erste Maxille (exp: enp $=1: 3$ ); zwei Haare an P5.

Art (2) rectanguläre Ecken am dritten Metasomasegment; Cilienquerreihen auf den Urosomsegmenten; Teilungsverhältnis des Mandibelpalps (4:1); erste Maxille (exp : enp = 1:3); Geschlechtsdimorphismus in $\mathbf{P 2}$.

Art (3) zwei dorsale Haare bei den Furcalästen; erste Maxille (exp : enp = 1:4); kurze Endklaue bei den zweiten Maxillen und den Maxillipeden; 


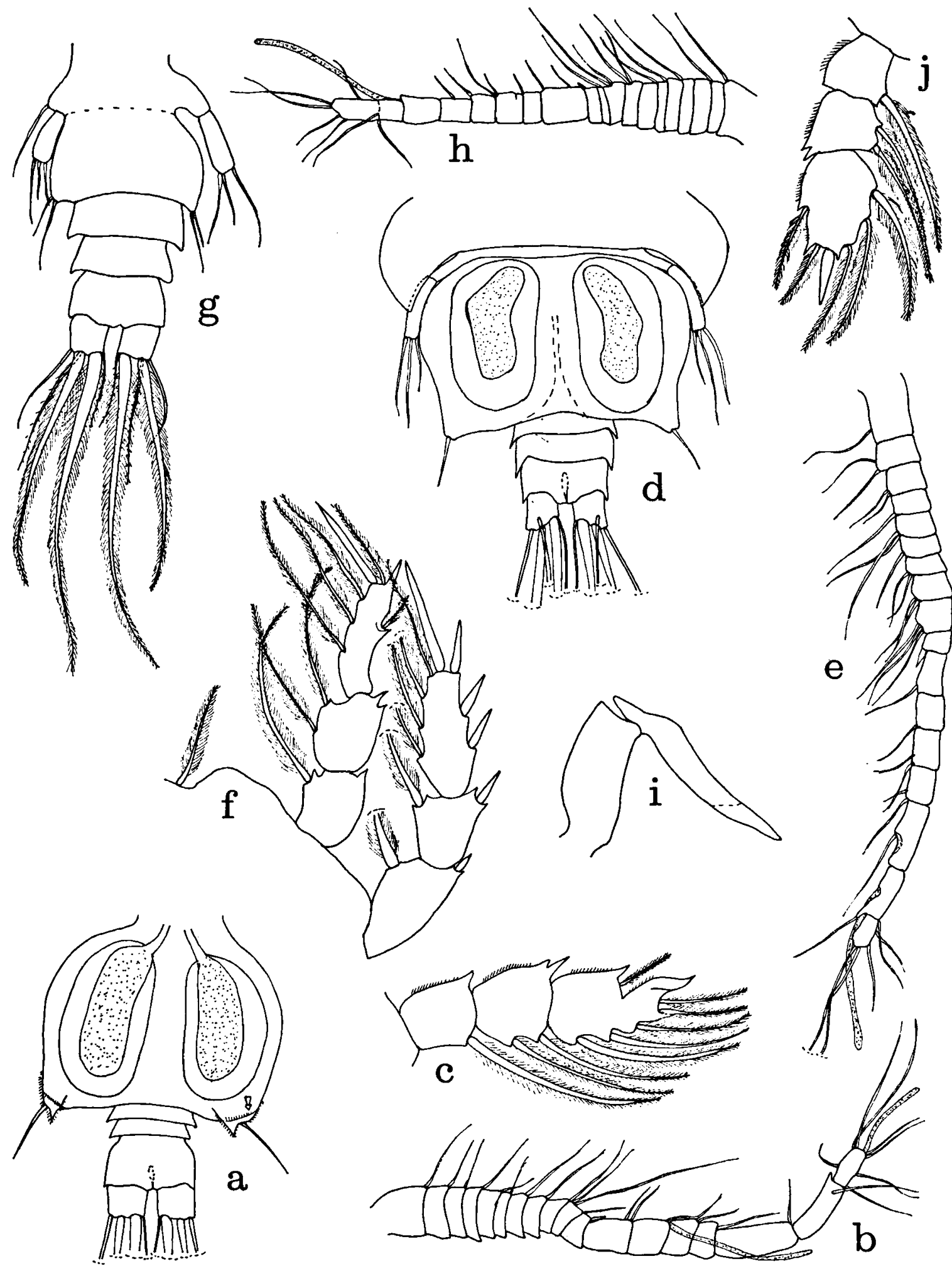

Fig. 5. Asterocheres complexus Stock 1960, Männchen $(a-c)$ : a, Urosom (Massstab II); b, erste Antenne (II); c, zweiter Ruderfuss, Innenast (III). Asterocheres mucronipes Stock, 1960, Männchen (d-f): d, Urosom (II); e, erste Antenne (II); f, dritter Ruderfuss (III). Asterocheres corneliae n. sp., Männchen (g-j): $\mathrm{g}$, Urosom (II); h, erste Antenne (II); i, zweite Maxille (III); j, dritter Ruderfuss, Innenast (III). 
die Fortsätze an den Ruderfüssen sind länger und mehr gebogen.

Art (4) Grösse: 0,83-0,93 mm; Zahn am Genitalsegment; zwei dorsale Haare bei den Furcalästen; Teilungsverhältnis des Mandibelpalps (3:1).

Art (5) das Analsegment ist viel länger; erste Maxille (exp : enp $=1: 4)$; nur zwei Haare an P5.

Art (6) Cilienquerreihen am Urosom; erste Maxille (exp : enp = 1:3); zwei Haare an P5.

Art (7) 18 Segmente an der ersten Antenne; Teilungsverhältnis des Mandibelpalps (4:1); erste Maxille (exp : enp $=1: 3$ ); die Endklauen der Maxillipeden sind stilettförmig und nicht gebogen.

Art (8) 19 Segmente an der ersten Antenne; Teilungsverhältnis des Mandibelpalps (3:1); erste Maxille (exp : enp $=1: 3$ ); die Fortsätze an den Ruderfüssen sind länger und gebogen.

Art (9) die Furca ist länger als das Analsegment; Teilungsverhältnis des Mandibelpalps (3:1); erste Maxille (exp : enp $=1: 3$ ).

Art (10) erste Maxille (exp : enp $=1: 5)$; zwei Haare an P5.

Die grösste Ubereinstimmung besteht mit $A$. complexus Stock, 1960. Die oben genannten Unterschiede jedoch lassen eine Identität nicht $\mathrm{zu}$. Eine enge Verwandtschaft ist jedoch nicht von der Hand zu weisen.

Der Name "corneliae" ist ein verspätetes Hochzeitsgeschenk an meine Frau Corry.

\section{Rhynchomyzon falco Giesbrecht, 1895}

\author{
Gesammeltes Material. - \\ 2\%, auf dem Schwamm Clathrina contorta (Bower- \\ bank), Les Trois Moines, $10-14 \mathrm{~m}, 2.8 .1965$.
}

Bisherige Funde. - Giesbrecht, 1895, Neapel; Wirt? und Sars, 1921, Norwegen; Wirt? Clathrina contorta ist der erste bekannte Wirt für Rhynchomyzon falco.

Die Färbung der gefundenen Tiere ist weiss.

\section{Acontiophorus antennatus Hansen, 1923}

\section{Gesammeltes Material. -}

19 , auf dem Schwamm Clathrina lacunosa (Johnston), Cap de l'Abeille, 20 m. 28.8.1961.

$1 \%$, auf dem Schwamm Oscarella lobularis (Schmidt), Cap Réderis, 20-24 m. 17.8.1962.
Hansen fand 1923 diese Art in der Davis-Strasse in grosser Tiefe (1435 Faden). Eiselt (1968) unterwarf Acontiophorus antennatus einer Uberprïfung. Beide geben die Grösse mit 1,23 mm an. Die vom Verfasser untersuchten Exemplare besitzen die Grösse von $0,82 \mathrm{~mm}$. Das würde besser mit den Grössenangaben von Acontiophorus scutatus Brady \& Robertson, 1873 übereinstimmen (0,90-1 mm; Giesbrecht, 1899: 104). Doch ist der Aussenast der zweiten Antenne zirka zwei Mal so lang wie das erste Innenastglied, und der Sipho erreicht nicht das Ende des Genitalsegments (Acontiophorus scutatus: Aussenast von A2 ist 0,8-1,2 Mal so lang wie das erste Innenastglied; der Sipho erreicht oder überragt die Furca). Das lässt eindeutig auf $A$. antennatus schliessen. Der beträchtliche Unterschied in den Grössen ist durch die Fundorte bedingt. Denn Lebewesen kälterer Regionen (Davis-Strasse, Norwegen) sind - wärmeregulatorisch bedingt - generell von grösserer Körperform als Lebewesen wärmerer Regionen.

Eiselt gibt in seiner Revision von $A$. antennatus (1968) auf Seite 180 die Borstenformel von P1 bis P4 an. Doch fehlen dabei einige Zahlen, die durch Fragezeichen ersetzt sind. Um diese Lücke zu füllen, sei hier die vollständige Borstenformel vermerkt:

\begin{tabular}{|c|c|c|c|}
\hline P 1 & $\begin{array}{l}\text { exp. } I-1 \\
\text { enp. } 0-1\end{array}$ & $\begin{array}{l}I-1 \\
0-2\end{array}$ & $\begin{array}{r}\mathrm{III}-\mathrm{I}-3 \\
1-5\end{array}$ \\
\hline P 2 & $\begin{array}{l}\text { exp. } I-1 \\
\text { enp. } 0-1\end{array}$ & $\begin{array}{l}I-1 \\
0-2\end{array}$ & $\begin{array}{r}\mathrm{III}-\mathrm{I}-4 \\
2-\mathrm{I}-3\end{array}$ \\
\hline P 3 & $\begin{array}{l}\text { exp. } I-1 \\
\text { enp. } 0-1\end{array}$ & $\begin{array}{l}\mathrm{I}-1 \\
0-3\end{array}$ & $\begin{array}{r}\mathrm{III}-\mathrm{I}-3 \\
1-\mathrm{I}-3\end{array}$ \\
\hline P 4 & $\begin{array}{l}\text { exp. } I-1 \\
\text { enp. } 0-1\end{array}$ & $\begin{array}{l}I-1 \\
0-2\end{array}$ & $\begin{array}{r}\mathrm{III}-\mathrm{I}-3 \\
1-\mathrm{I}-2\end{array}$ \\
\hline
\end{tabular}

Der Wirt von $A$. antennatus war bisher noch nicht bekannt. Clathrina lacunosa und Oscarella lobularis sind daher die ersten bekannten Wirte.

Eiselt sagt über $\boldsymbol{A}$. antennatus abschliessend: "Obwohl die Vermutung nahe liegt, dass Acontiophorus antennatus eine vikariierende Subspecies oder gar nur eine modifikatorisch geprägte, in grossen Tiefen lebende Variante von $A$. scutatus sei, muss eine solche Entscheidung einstweilen zurückgestellt werden, da das vorhandene Material von $A$. antennatus nicht ausreicht und aus dem sich über 45 Längengrade erstreckenden Raum zwischen den Faröern und der Davis-Strasse $\mathrm{m}$. W. Fänge von $\boldsymbol{A}$. scutatus nicht gemeldet worden sind."

In dieser Arbeit nun wird $A$. antennatus als selbständige Species angeführt. Sollte sich später 

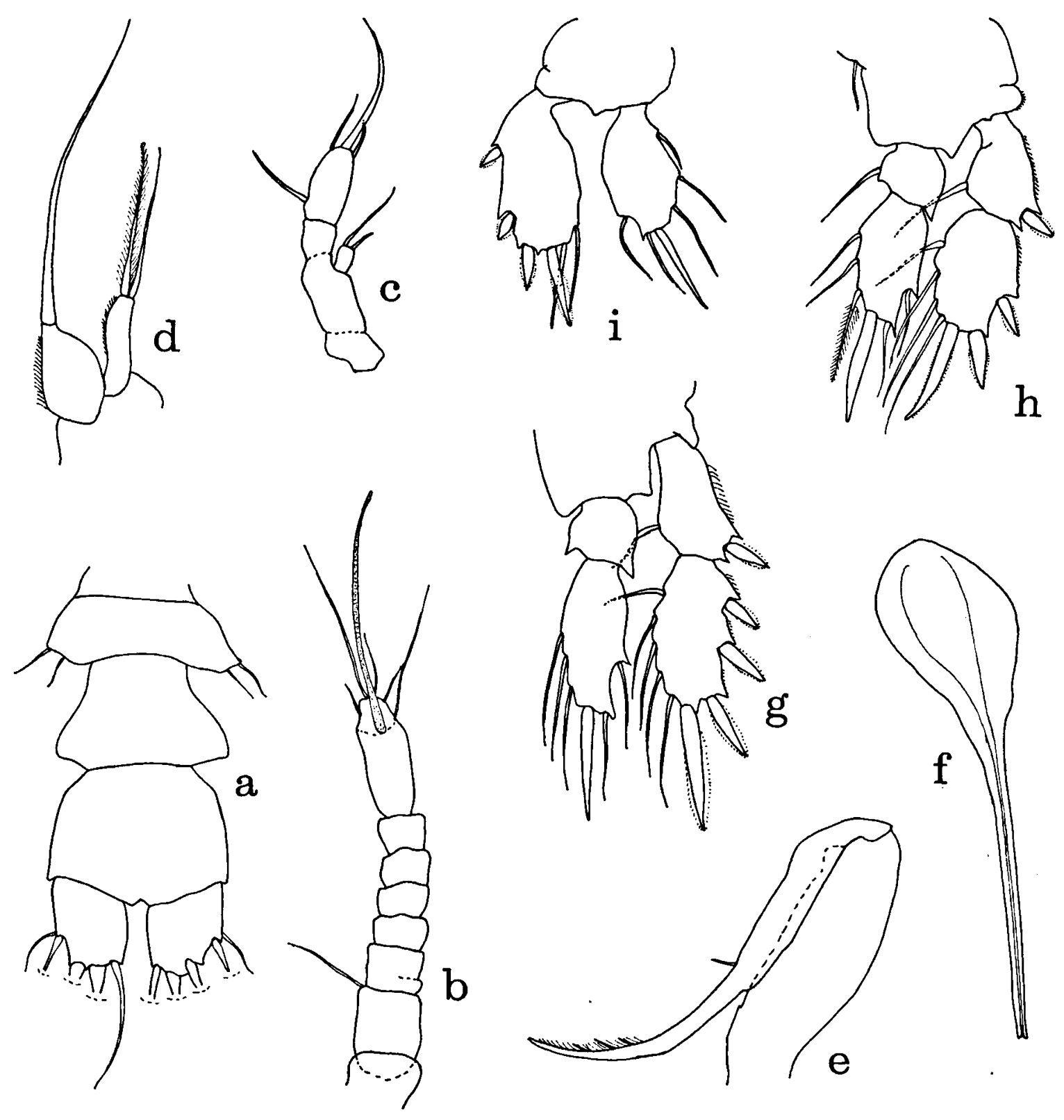

$\mathrm{h}$

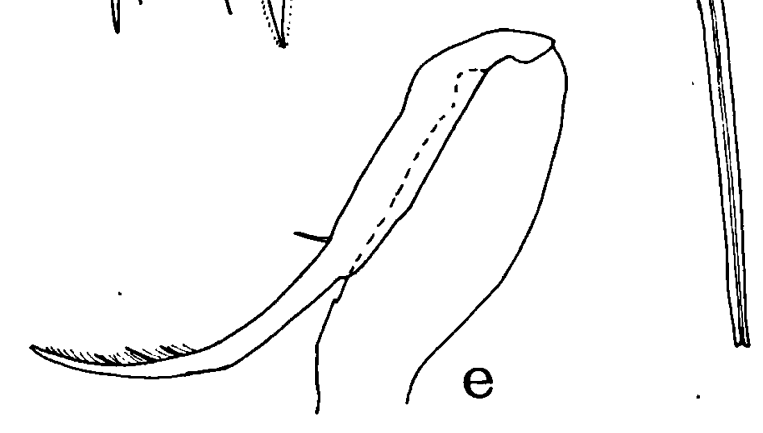

Maßstäbe :

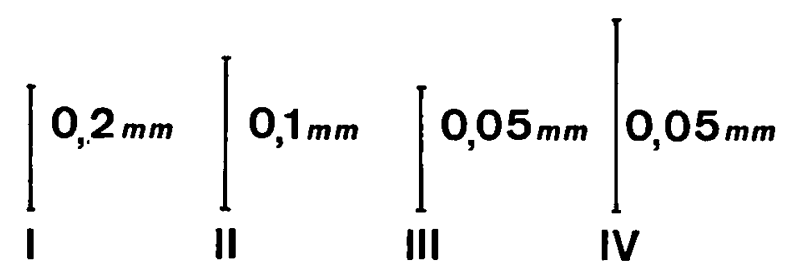

Fig. 6. Myzopontius sp., Copepodid-Stadium: a, Urosom (Massstab III); b, erste Antenne (III); c, zweite Antenne (III); d, erste Maxille (III); e, zweite Maxille (III); f,

Sipho (III); g, zweiter Ruderfuss (III); h, dritter Ruderfuss (III); i, vierter Ruderfuss (III). 
eine eindeutige Klarstellung der Grenzen ergeben, so will ich mich dieser gerne beugen. Vielleicht ist dieser Fund ein nützlicher Beitrag dazu.

Verbreitung: Davis-Strasse (Hansen), Banyuls (Schirl).

10. Myzopontius pungens Giesbrecht, 1895

Myzopontius pungens Giesbrecht, 1895; Sars, 1915.

Gesammeltes Material. -

$18 \%, 6 \hat{\sigma}$, auf dem Schwamm Clathrina primordialis (Schmidt), Cap Oullestreil, $\pm 10 \mathrm{~m}$. 26.8.1962.

13 \% , 1 ơ , derselbe Wirt, Pier Banyuls, $10-12$ m. 27.7. 1963.

2 \&, 1 s, derselbe Wirt, Cap de l'Abeille, 10-17 m. 30.7.1963.

9\%, $21 \mathrm{\delta}$, auf dem Schwamm Clathrina contorta (Bowerbank), Les Trois Moines, 10-14 m. 2.8.1965.

Giesbrecht fand Myzopontius pungens im Golf von Neapel, ohne den Wirt zu kennen. Th. Scott konnte beim Franz-Josefs Land Exemplare finden, Sars an der Südküste von Norwegen. Brian meldet Funde von der Insel St. Catharina und Canal di Leme (Rovinj, Jugoslavien) in der Tiefe von 1-3 m, doch ebenfalls ohne Wirtangabe. Nun sind zahlreiche Exemplare auf Clathrina primordialis und Clathrina contorta gefunden worden. Beide Schwämme, sicher aber Clathrina contorta, sind typische Wirte für Myzopontius pungens.

Die Farbe der auf Clathrina contorta gefundenen Tiere war bei den kleineren Exemplaren durchscheinend weiss, bei den grösseren durchscheinend orange bis bräunlich. Die Farbe der Augen reicht von leicht rötlich auf aprikosenfärbig.

\section{Myzopontius sp.}

Gesammeltes Material. -

42 Exemplare vom Schwamm Clathrina primordialis (Schmidt), Cap Oullestreil, $\pm 10 \mathrm{~m}, 26.8 .1962$.

4 Exemplare, derselbe Wirt, Cap de l'Abeille, 10-17 m, 30.7.1963.

1 Exemplar vom Schwamm Clathrina contorta (Bowerbank). Les Trois Moines, 10-14 m, 2.8.1965.

Mit grosser Wahrscheinlichkeit handelt es sich hier um Entwicklungsstufen von Myzopontius pungens. Dafür spricht vor allem die zweite Maxille. Deren Endklaue trägt im letzten Teil eine Reihe von kleinen Cilien, ungefähr zwanzig Stück. Sowohl bei $M$. australis, als auch bei $M$. innomi- natus finden wir davon maximal fünf bis sechs. Weiters liegt es nahe, dass die Jugendformen zur selben Art gehören, woraus der grösste Teil der Probe besteht, nämlich $M$. pungens, und keine anderen Vertreter der Myzopontius Arten angetroffen wurden.

Drei Exemplare davon befanden sich noch in einem sehr frühen Entwicklungsstadium, so dass es mir der Mühe wert schien, davon einige Abbildungen anzufertigen. Die Grösse betrug $0,41 \mathrm{~mm}$. Das Urosom (Fig. 6a) ist noch zweigliedrig und sehr undifferenziert. Die erste Antenne (Fig. 6b) besitzt erst acht Glieder, doch sind zwei folgende Trennungsschritte schon erkennbar. Die zweite Antenne (Fig. 6c) ist bereits sehr gut entwickelt; ebenso besitzen schon die erste Maxille (Fig. 6d), die zweite Maxille (Fig. 6e) und der Sipho (Fig. 6f) ihre endgültige Form. P1 bis P3 (Fig. $6 \mathrm{~g}$, h) sind noch zweigliedrig, P4 (Fig. 6i) ist noch eingliedrig.

\section{Pteropontius cristatus Giesbrecht, 1895}

Gesammeltes Material. -

19, von dem Schwamm Clathrina clathrus (Schmidt), Pier Banyuls, $\pm 10 \mathrm{~m}$. 2.8.1962.

$1 \%, 1 \hat{\delta}$, von dem Schwamm Clathrina primordialis (Schmidt), Cap Oullestreil, $\pm 10 \mathrm{~m}$. 26.8.1962.

Giesbrecht fand Pteropontius cristatus in Neapel, ohne den Wirt zu kennen. Brian sammelte sein Material auf Bagnole (Rovinj) zwischen Algen in 1,5 m Tiefe. Stock konnte 1965 bei Le Troc (Banyuls) 2 ㅇ 9 und 10 auf Hymeniacidon breviscuspis (Topsent) finden. Clathrina clathrus und Clathrina primordialis sind demzufolge neue Wirte für Pteropontius cristatus.

\section{BESPRECHUNG DER EINZELNEN WIRTE}

\section{C a 1 c is pong a e}

Clathrina clathrus (O. Schmidt) (=Leucosolenia cl.): "Geflecht 0,5-1 mm starker Röhren, Kolonien bis $10 \mathrm{~cm}$, lebhaft gelb, mit Triactinen aus konisch-zyl., im Umriss unregelmässigen Strahlen; meist im Halbschatten der Grotten, in 1-3 m Tiefe, aber nicht häufig" (Fauna und Flora der Adria, R. Riedl, 1963).

Fünf Proben von Clathrina clathrus standen mir zur Verfügung. Die zahlenmässige Verteilung der Tiere, siehe Tabelle I. 


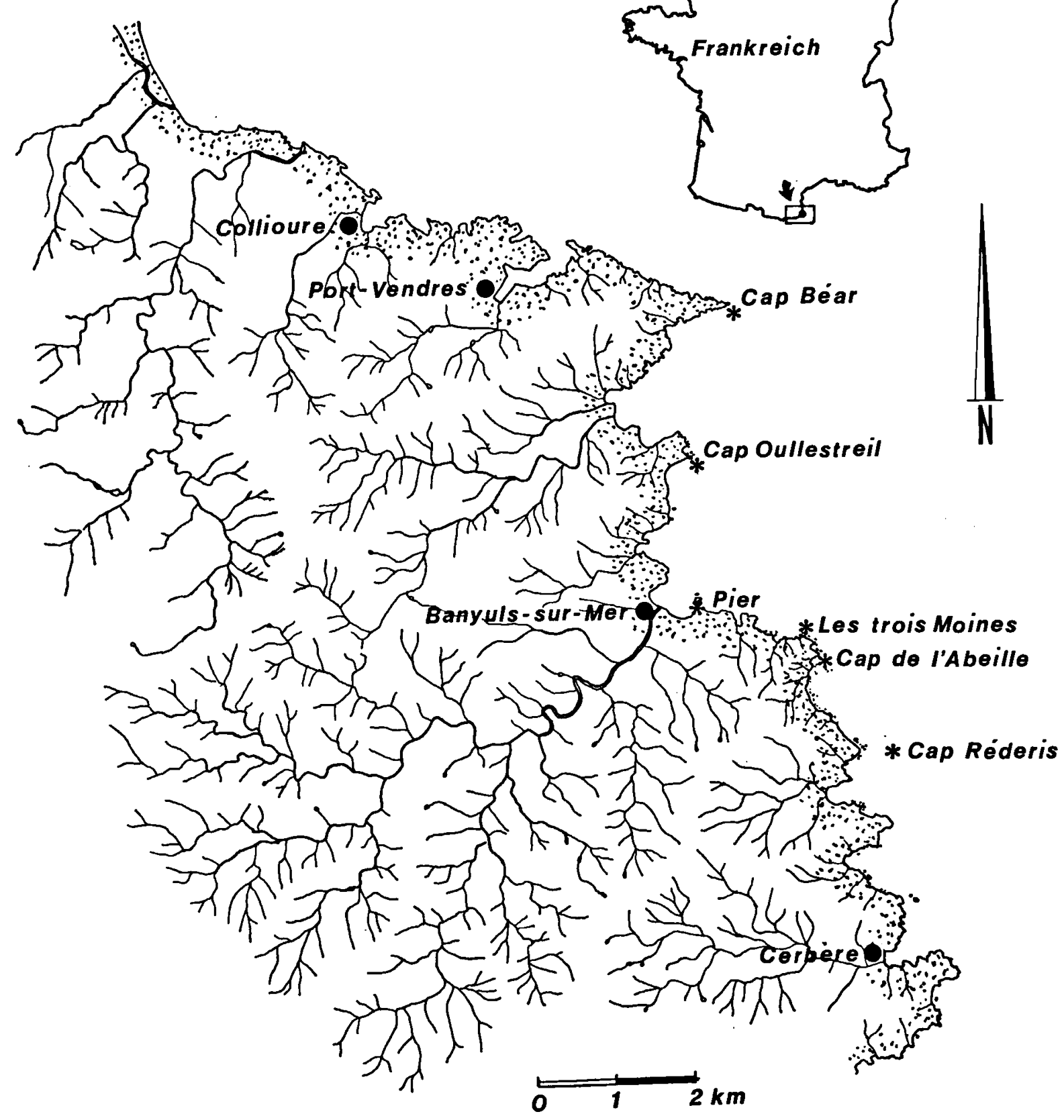

Fig. 7. Die lage der im Text genannten Fundorte in der Nähe von Banyuls (Frankreich, Pyrénées-Orientales). 
Tabelle I
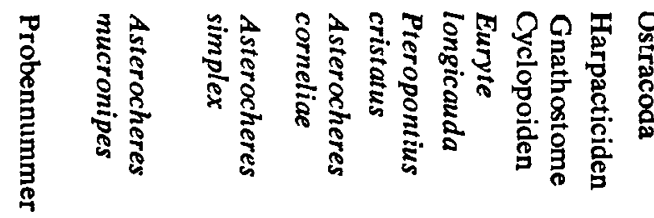

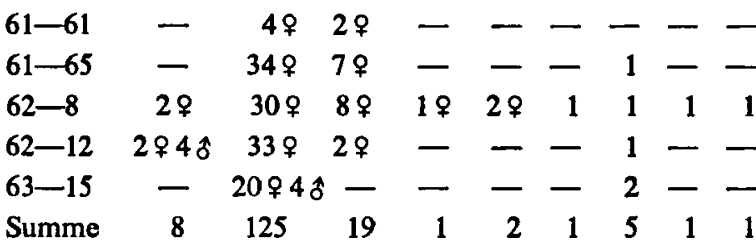

Die prozentuelle Verteilung der siphonostomen Cyclopoiden auf Clathrina clathrus ist wiedergegeben in Fig. 8.

Insgesamt wurden 153 siphonostome Cyclopoiden auf Clathrina clathrus gefunden. Wie aus den oben gezeigten Tabellen ersichtlich ist, herrscht Asterocheres simplex mit 125 Exemplaren vor. Offensichtlich handelt es sich hier um einen typischen Wirt von Asterocheres simplex.

Mit der Bezeichnung "typischer Wirt" schliesse ich mich mehreren anderen Autoren an (Stock; Bresciani \& Lützen) und will damit unterstreichen, dass eine spezielle Bevorzugung eines bestimmten Wirtes nicht von der Hand zu weisen ist.

Die Vertreter der anderen gefundenen Entomostraca habe ich der Vollständigkeit halber auf die Tabelle gesetzt, doch will ich sie bei der Besprechung ausser Acht lassen, da die Materie sonst zu unübersichtlich würde.

Clathrina lacunosa (Johnston): gestielter Vertreter der Clathrina Arten.

Drei Proben von Clathrina lacunosa wurden untersucht (siehe Tabelle II).
Tabelle II
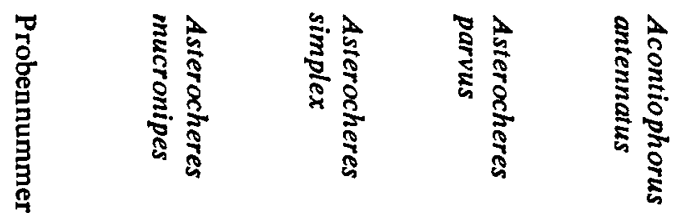

$\begin{array}{lcc}62-3 & - & 5 q \\ 61-52 & 5928 & 3 q \\ 61-71 & - & 5 q \\ \text { Summe } & 7 & 13\end{array}$

$$
\frac{1 \hat{0}}{-}
$$$$
1 \text { 우 }
$$$$
\overline{1}
$$

Die Verteilung, in Prozenten ausgedrückt, findet man in Fig. 9.

Von den 22 gefundenen Tieren umfasst wieder Asterocheres simplex den grössten, prozentuellen Anteil (58\%). Daher kann man wieder von einem typischen Wirt sprechen; sein grosser Gegenspieler (Asterocheres mucronipes mit 32\%) befindet sich hier sicher nicht auf seinem typischen Wirt, wie später gezeigt wird. Asterocheres parvus und $A$ contiophorus antennatus bilden mit je einem Vertreter eine verschwindende Minderheit und gehören auch, was aus den vorhandenen Literaturangaben ersichtlich ist, zu selten und in geringer Anzahl gefundenen Vertretern der Asterocheridengruppe.

Clathrina primordialis (Mont.) (= Leucosolenia coriacea): "Flache bis polsterförmige Netze aus 0,5-1 mm starken Röhren, Kolonie bis $10 \mathrm{~cm}$, gewöhnlich weiss, mit Triactinen aus - kon. Strahlen. Häufig bis massenhaft in Löchern und Kleinhöhlen, im sauberen, bewegten Wasser" (Fauna und Flora der Adria, R. Riedl, 1963).

Sechs Proben von Clathrina primordialis lagen vor (Tabelle III).

Tabelle III
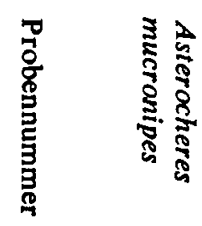

$\begin{array}{lcc}61-70 & - & 7 \% \\ 62-9 & - & 8 \% \\ 62-36 & 1 \% & 3 \% \\ 63-17 & - & 9 \% \\ 63-22 & 1 \% & 19 \% 1 \% \\ 63-23 & - & 1 \% \\ \text { Summe } & 2 & 48\end{array}$

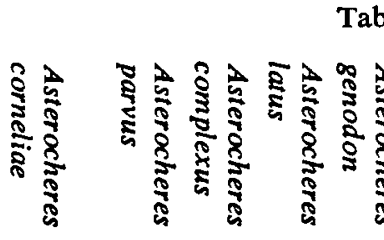

$$
7938
$$
182 ㅇ
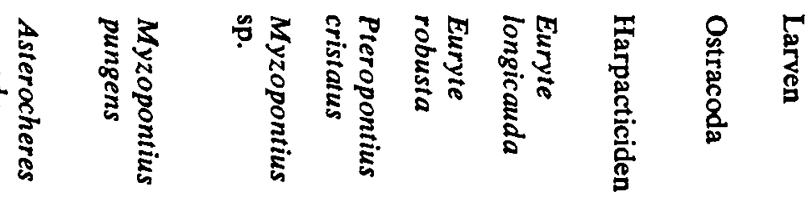

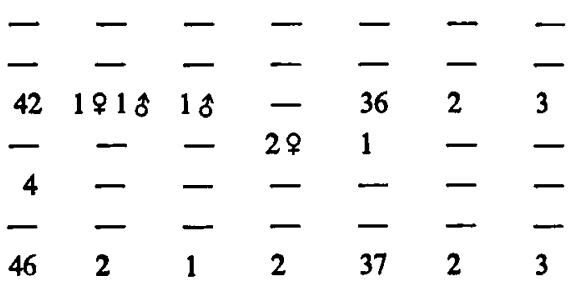



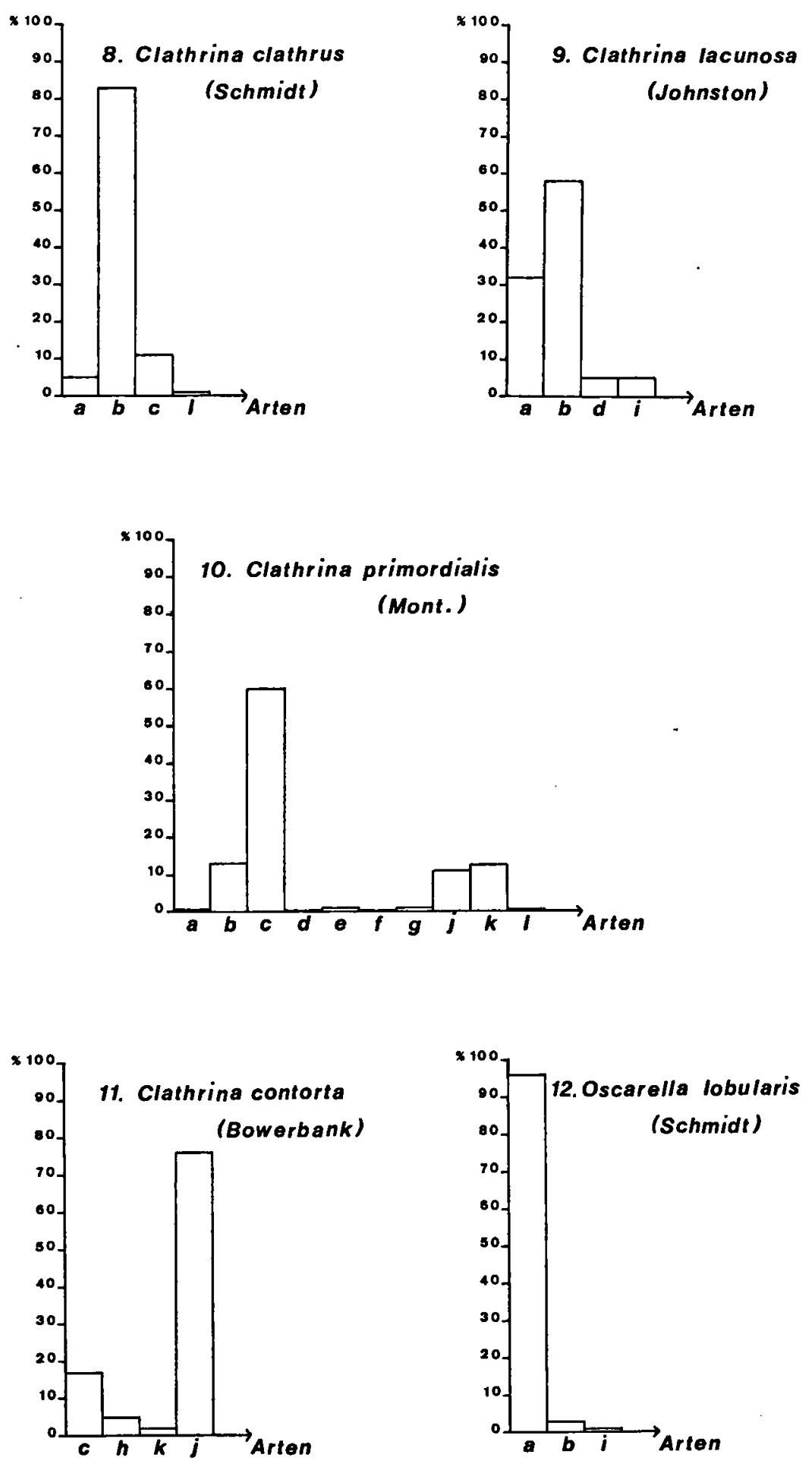

Fig. 8-12. Die prozentuelle Verteilung der siphonostomen Cyclopoiden auf vier Clathrina Arten und auf $O s$ carella lobularis.

a, Asterocheres mucronipes; b, A. simplex; c, A. corne- liae; d, A. parvus; e, A. complexus; f, A. latus; g, $A$. genodon; h, Rhynchomyzon falco; i, Acontiophorus antennatus; j, Myzopontius pungens; k, Myzopontius sp.; 1, Pteropontius cristatus. 
Die prozentuelle Verteilung wird in Fig. 10 gegeben.

Die Verteilung der Tiere auf Clathrina primordialis ist nicht so eindeutig. In vier Proben herrscht Asterocheres corneliae vor (61-70: 58\%; 62-9: 55\%; 62-36: 71\%; 63-23: 66\%), in der Probe 63-17 Myzopontius pungens mit 60\% und in der Probe 63-22 Asterocheres simplex mit 49\%. Von den 365 auf Clathrina primordialis gefundenen Exemplaren siphonostomer Cyclopoiden sind 215 von der Art Asterocheres corneliae. Doch in einer Probe (63-17) ist kein einziges Exemplar zu finden, und nur in vier Proben erreicht $A$. corneliae den höchsten Prozentsatz. Trotzdem ist anzunehmen, dass Clathrina primordialis ein typischer Wirt für Asterocheres corneliae ist. Für Myzopontius pungens ist nicht so sehr die Häufigkeit auffallend, wohl aber, dass $M$. pungens allein hier und auf Clathrina contorta, einer ebenfalls weissen Clathrina Art, gefunden worden ist. Es ist anzunehmen, dass Clathrina primordialis, trotz des nicht allzu hohen prozentuellen Anteils, ein typischer Wirt für diese Myzopontius Art ist. Die grosse Streuung der anwesenden Parasiten (11 Arten) ist wohl durch das "häufige bis massenhafte" Vorkommen von Clathrina primordialis bedingt.

Clathrina contorta (Bowerbank): 'Krustenförmige Netze aus 0,50-1 mm starken Röhren, Kolonien bis $2 \mathrm{~cm}$, Farbe weisslich, mit Triactinen und Tetractinen aus konischen Strahlen, oft auch mit Oxen. Häufig in gut beströmten Höhlenteilen, unter Steinen, sowie auf Posidonia-Rhizoiden und Cystoseira-Strünken, in 1-10 m Tiefe". (Fauna und Flora der Adria, R. Riedl, 1963).

Leider stand nur eine einzige Probe dieses Schwammes zur Verfügung (Tabelle IV).

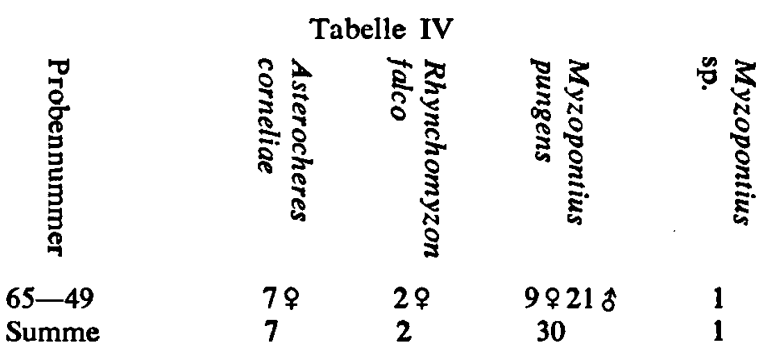

Prozentueller Anteil, siehe Fig. 11.

Von den vierzig gefundenen Tieren gehören dreissig, das sind 76\%, der Art Myzopontius pungens an. Offensichtlich ein typischer Wirt für $M y$ zopontius pungens.

Uber die Clathrina Arten kann abschliessend fol- gendes gesagt werden: man kann eine deutliche Trennlinie zwischen die vier untersuchten Arten legen. Clathrina clathrus und C. lacunosa stehen auf der einen, $C$. primordialis und $C$. contorta auf der anderen Seite. Auf den beiden ersten Schwämmen ist Asterocheres simplex mit seinem kurzen, kräftigen Sipho vorherrschend, auf Clathrina primordialis und $C$. contorta kommen häufig Myzopontius Arten, Asterocheres corneliae und andere Vertreter mit langem Sipho vor. Leider fehlt mir die dazu nötige Zeit und die entsprechende Kenntnis über Schwämme, um die Ursachen zu beleuchten, doch bin ich fest davon überzeugt, dass eine Beziehung zwischen dem Wirt und der Morphologie der parasitierenden Cyclopoiden besteht. Clathrina clathrus und C. lacunosa sind eher seltene Schwämme, $C$. primordialis und $C$. contorta eher häufig zu finden, wodurch die grosse Artenfülle darauf erklärbar ist.

De mospong i a

Oscarella lobularis (O. Schmidt):

"fleischig weiche, lappige Krusten, braun, seltener blau; ziemlich regelmässig auf Posidonia-Rhizoiden" (Fauna und Flora der Adria, R. Riedl, 1963), weiter häufig auf Kalkalgenboden.

Die zwei mir vorliegenden Proben ergeben folgendes Verteilungsbild (Tabelle V).

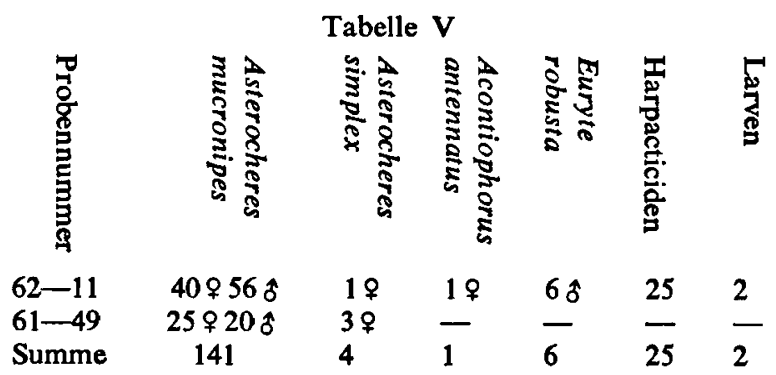

Die Anteile in Prozenten ausgedrückt, siehe Fig. 12.

Wenn sonst nirgends, so ist hier sicher eine Do. minanz vorliegend. Von den insgesamt 146 gefundenen Tieren gehören 141 der Art Asterocheres mucronipes an. A. mucronipes mit seinem langen und zarten Sipho scheint der prädestinierte Gast für den "fleischig weichen, lappigen Krustenschwamm" Oscarella lobularis zu sein.

"Wie die breiten, mit Hakenzähnen besetzten Mandibelladen die Pontelliden als Räuber verraten würden, auch wenn man in ihrem Darm nicht 
die Chitinreste anderer Entomostracen fände, so sind der Sipho der Asterocheriden und die darin liegenden Mandibelstilette ein Beweis dafür, dass die Arten dieser Familie ihre Nahrung durch Anstechen anderer Organismen und durch Saugen von deren Säften gewinnen. Zugleich aber entnimmt man aus ihren wohlentwickelten Schwimmfüssen und aus der Schnelligkeit, mit der sie das Wasser durcheilen können, dass sie leicht im Stande sind, ihr Opfer zu verlassen und ein neues zu suchen. Die Ernährungsweise $\operatorname{der}$ A. hat also eine grosse Ähnlichkeit mit derjenigen so mancher Insekten, die eine rein parasitische Ernährung mit einer hoch entwickelten Locomotionsfähigkeit verbinden." (Giesbrecht, 1899: 199, 1. Absatz). Die grosse Lokomotionsfähigkeit der meisten Vertreter dieser parasitischen Copepodengruppe ist sicher nicht von der Hand zu weisen. Doch versuchte ich mit Hilfe des von mir untersuchten Materials nachzuweisen, dass eine Dominanz einzelner Arten auf bestimmten Wirten besteht. Um einen grösseren UUberblick zu geben, bemühte ich mich, aus den vorhandenen Literaturangaben eine Liste der Wirte mit ihren Parasiten und deren Anzahl zusammenzustellen. Leider fehlen nur allzuoft die genauen Zahlenangaben.

\section{WIRT}

1) Algen und Seegräser:

a) flottierende, gelatinöse Alge

b) Grünalgen:

Codium arabicum

c) Braunalgen:

Laminaria sp.

Laminaria saccharina

Cystoseira

Sargassum

d) Rotalgen, gen. et sp.

e) Seegras:

Posidonia

2) Kalkschwämme:

Clathrina clathrus

Clathrina lacunosa

Clathrina primordialis

Clathrina contorta

Pericharax heteroraphis
GAST

Collocheres gracilipes

Myzopontius australis

Dermatomyzon nigripes

Dermatomyzon nigripes

Asterocheres intermedius Acontiophorus scutatus

Dyspontius striatus

Asterocheres latus

Dyspontius striatus

Asterocheres violaceus

Asterocheres mucronipes

Asterocheres simplex

Asterocheres corneliae

Pteropontius cristatus

Asterocheres mucronipes

Asterocheres simplex

Asterocheres parvus

Acontiophorus antennatus

Asterocheres mucronipes

Asterocheres simplex

Asterocheres corneliae

Asterocheres parvus

Asterocheres complexus

Asterocheres latus

Asterocheres genodon

Myzopontius pungens

Myzopontius sp.

Pteropontius cristatus

Asterocheres corneliae

Rhynchomyzon falco

Myzopontius pungens

Myzopontius sp.

Asterocheres proboscideus
ANZAHL

$\begin{array}{cl}- & \text { Canu, 1893 } \\ 1 \% & \text { Stock, 1966a } \\ - & \text { Brady, 1880 } \\ 1 \% & \text { Lang, 1949 } \\ - & \text { Brian, 1923 } \\ - & \text { Brian, 1923 } \\ 5 \% & \text { Brian, 1923 } \\ 2 \% 2 \delta^{\circ} & \text { Lang, 1949 } \\ - & \text { Lang, 1949 } \\ & \text { Brian, 1923 }\end{array}$

4 우 $4 \hat{\circ}$

$12194 \hat{\delta}$

19 \%

19

$5 \% 28$

$13 \%$

1 is

1 운

1918

47 웅

$21293 \hat{\delta}$

$1 \hat{s}$

5 oิ

19

4 앙

33 क 8 के

$46 \mathrm{Ex}$.

$191 \%$

79

$2 \%$

9 \%21

$1 \mathrm{Ex}$.

7 우

\section{QUELLE}

\author{
Canu, 1893 \\ Lang, 1949 \\ Brian, 1923 \\ Brian, 1923 \\ Brian, 1923 \\ Lang, 1949 \\ Brian, 1923
}

Schirl, 1973

Schirl, 1973

Schirl, 1973

Schirl, 1973

Schirl, 1973

Schirl, 1973

Schirl, 1973

Schirl, 1973

Schirl, 1973

Schirl, 1973

Schirl, 1973

Schirl, 1973

Schirl, 1973

Schirl, 1973

Schirl, 1973

Schirl, 1973

Schirl, 1973

Schirl, 1973

Schirl, 1973

Schirl, 1973

Schirl, 1973

Schirl, 1973

Stock, 1966a

3) Kieselschwämme:

a) Homosclerophorida: ohne Skelett, ohne Spongin

Oscarella lobularis 
WIRT

Oscarella sp.

b) Hadromerina:
GAST

Asterocheres mucronipes

Suberites domuncula

Cliona sp.

Tethya aurantia

Asterocheres echinicola

Asterocheres suberites

Asterocheres echinicola

Stenopontius humesi

Asterocheres sp.
Suberites sp.

Cliona celata

c) Halichondrina:

Halichondria sp.

Halichondria bowerbanki

Halichondria panicea

Hymeniacidon brevicuspis

d) Poecilosclerina: mit Nadeln und Sponginfasern

Asterocheres jeanyeatma-

Acontiophorus brevifurca-

Mycale mytillorum

Hemimycale columella

Crella elegans

Agelas oriodes

e) Haplosclerina: mit Nadeln und Sponginfasern

Calyx nicaeensis

Haliclona oculata

Haliclona sp.

Haliclona permollis

Haliclona indistincta

Petrosia ficiformis

Siphonochalina communis

Reniera sp.

Reniera cinerea

Microciona prolifera

Craniella gravida

Grantia compressa

Chalina oculata

Sigmatoxella annexa

Ulosa stuposa

Octavella galangaui

Ciocalypta penicillis

4) Hornschwämme:

Spongelia fragilis var. ramosa

Verongia aerophoba

Verongia cavernicola

Ircinia muscarum

Dysidea tupha Asterocheres parvus
Sponginfasern

Asterocheres parvus nae

Cryptopontius gracilis

Asterocheres echinicola

Asterocheres echinicola

Asterocheres parvus

Scottocheres elongatus

Asterocheres halichondriae tus

Pteropontius cristatus

Sestropontius bullifer

Scottocheres laubieri

Asteropontius mycalei

Pteropontius cristatus

Cryptopontius capitalis

Scottocheres laubieri

Cryptopontius capitalis

Asterocheres echinicola

Asterocheres genodon

Cryptopontius gracilis

Apodomyzon brevicorne

Apodomyzon longicorne

Cryptopontius thorelli

Cryptopontius capitalis

Asterocheres genodon

Asterocheres renaudi

Asterocheres parvus

Scottocheres elongatus

Asterocheres jeanyeatmanae

Cryptopontius gracilis

Cryptopontius gracilis

Asterocheres echinicola

Asterocheres echinicola

Asterocheres boecki

Scottocheres elongatus

Scottocheres elongatus

Cryptopontius capitalis

Asterocheres echinicola

Asterocheres complexus Acontiophorus scutatus

Cryptopontius minor

Entomolepis adriae

Entomolepis adriae

Psilomyzon pauciseta

Scottocheres elongatus

Tuphacheres micropus
ANZAHL

197

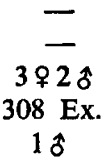

viele ㅇ $u . \delta$
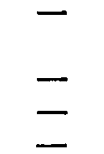

69 웅

9 की

$47 \% 3 \hat{s}$

1 ㅇ 1 Cop.

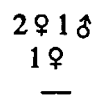

49

1 oे 1 Cop.

1 우

\section{QUELLE}

Stock, 1966a

Th. Scott, 1898

Giesbrecht, 1897

Stock, 1960

Murnane, 1967

Stock, 1960

Lang, 1949

Yeatman, 1970

Yeatman, 1970

Hamond, 1968

Stock, 1967

Klie, 1933

Klie, 1933

Stock, 1966a

Stock, 1966a

Stock, 1965

Stock, 1965

Stock, 1967

Krishnaswami, 1956

Stock, 1965

Stock, 1965

Stock, 1967

\begin{tabular}{|c|c|}
\hline 19 & Stock, 1965 \\
\hline & Stock, 1967 \\
\hline $8 \%$ & Stock, 1966a \\
\hline 一 & Yeatman, 1970 \\
\hline 3918 & Stock, 1970 \\
\hline $3 \%$ & Stock, 1970 \\
\hline $1 \hat{\delta}$ & Stock, 1965 \\
\hline 19 & Stock, 1965 \\
\hline 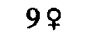 & Stock, 1966b \\
\hline- & Canu, 1891 \\
\hline- & Klie, 1933 \\
\hline 一 & Klie, 1933 \\
\hline - & Yeatman, 1970 \\
\hline- & Yeatman, 1970 \\
\hline- & Yeatman, 1970 \\
\hline- & Stock, 1967 \\
\hline - & Th. Scott, 1893 \\
\hline 一 & Th. Scott, 1893 \\
\hline & Stock, 1967 \\
\hline & Stock, 1967 \\
\hline 18 & Stock, 1965 \\
\hline & Hamond, 1968 \\
\hline
\end{tabular}

2 ㅇ Cop.

19

19

19

sehr viele Ex.

viele Ex.

22 ㅇ 15 oै

-

$14 \% 17$ oิ
Stock, 1960

Stock, 1960

Stock, 1960

Stock, 1965

Stock, 1965

Stock, 1965

Stock, 1965

Stock, 1967

Stock, 1965 


\section{WIRT}

5) Anthozoa:

Anemonia sulcata

Rhodactis rhodostoma

Pocillopora damicornis f. favosa

Pocillopora damicornis

Montipora sp.

Stylophora cf. erythrea

Stylophora pistillata

Stylophora subseriata

Porites sp.

Echinopora lamellosa

Platygyra sp.

Alcyonaria gen. et. sp.

Paraerythropodium coralloides

Leptogorgia sarmentosa

Dendrophyllia nigrescens

Dendrophyllia micranthus

Dendrophyllia sp.

Eunicella stricta

6) Mollusca:

Doris sp.

Nudibranchia gen. et sp.

Chlamys opercularis

7) Crinoidea:

Antedon mediterranea

Heterometra savignyi

Oligometra serripinna

8) Holothuroidea:

Holothuria tubulosa

Holothuria stellati

Stichopus chloronotus

Stichopus regalis

Stichopus tremulus

Isostichopus badionotus

Brandtothuria arenicola

Semperothuria surinamensis

Microthele parvula

9) Echinoidea:

Calveriosoma gracile

Calveria gracilis

Sperosoma quincunciale

Hygrosoma hoplacantha

Hygrosoma petersi

Arbacia lixula

Sphaerechinus granularis

Echinus esculentus
GAST

Dinopontius acuticauda

Asterocheres scutatus

Asteropontius corallophilus

Monocheres mauritianus

Asteropontius corallophilus

Asteropontius corallophilus

Asteropontius corallophilus

Asteropontius corallophilus

Asteropontius corallophilus

Pteropontius pediculus

Bradypontius pichoni

Asterocheres indicus

Acontiophorus bracatus

Acontiophorus bracatus

Cholomyzon palpiferum

Cholomyzon palpiferum

Cholomyzon palpiferum

Acontiophorus bracatus

Asterocheres mucronipes

Artotrogus orbicularis

Artotrogus orbicularis

Scottocheres elongatus

Collocheres gracilicauda

Collocheres uncinatus

Collocheres uncinatus

Allantogynus delamarei

Allantogynus delamarei

Nanaspis tonsa

Nanaspis mediterranea

Nanaspis nanae

Nanaspis pollens

Nanaspis exigua

Nanaspis media

Nanaspis pollens

Nanaspis truncata

Nanaspis pollens

Nanaspis truncata

Nanaspis truncata

Calvocheres globosus

Calvocheres globosus

Calvocheres globosus

Calvocheres engeli

Calvocheres oblongus

Asterocheres violaceus

Asterocheres violaceus

Asterocheres minutus

Asterocheres violaceus

Asterocheres violoceus

Asterocheres violaceus

Asterocheres echinicola

Asterocheres violaceus

Asterocheres violaceus
ANZAHL

6 웅수

29

3 웅

19

$995 \delta$

2 운

29

19

19

18

1 ㅇ

1 운

29

39

$38 \% 26$

7 के 4 \%

7978

5958

4 운
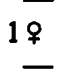

$41 \% 11$ \%

4 앙

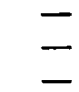

$10 \% 8$ क

sehr viele Ex.

$156 \% 139$ \&

30 운중

27 웅

einige Ex.

viele Ex.

einige Ex.

viele Ex.

14 \%20 ठ

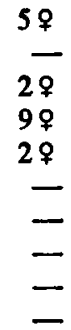

viele ㅇ $\mathrm{u} . \diamond$

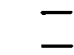

viele Ex.

\section{QUELLE}

Stock, 1960

Stock, 1966b

Stock, 1966a

Stock, 1966a

Stock, 1966a

Stock, 1966a

Stock, 1966a

Stock, 1966a

Stock, 1966a

Stock, 1966a

Stock, 1966a

Sewell, 1949

Stock \& Kleeton, 1963

Stock \& Kleeton, 1963

Stock \& Humes, 1969

Stock \& Humes, 1969

Stock \& Humes, 1969

Stock \& Kleeton, 1963

Stock, 1960

Boock, 1859

Lang, 1949

Norman \& Scott, 1906

Rosoll, 1888

Stock, 1966

Stock, 1966

Changeux, 1958

Changeux, 1958

Humes \& Cressey, 1959

Stock \& Kleeton, 1962

Bresciani \& Lützen, 1962

Stock, Humes \& Gooding, 1962

Stock, Humes \& Gooding, 1962

Stock, Humes \& Gooding, 1962

Stock, Humes \& Gooding, 1962

Stock, Humes \& Gooding, 1962

Stock, Humes \& Gooding, 1962

Stock, Humes \& Gooding, 1962

Stock, Humes \& Gooding, 1962

Stock, 1968

Hansen, 1902

Stephensen, 1935

Stock, 1968

Stephensen, 1935

Stock, 1960

Giesbrecht, 1897

Giesbrecht, 1897

Bocquet, 1952

Bocquet, Stock \& Louise, 1963

Gooding, 1957

Sars, 1915

Sars, 1915

Bresciani \& Lützen, 1962 


\section{WIRT}

Echinus esculentus

Echinus elegans

Echinus microtuberculatus

Psammechinus microtuberculatus

Psammechinus miliaris

Paracentrotus lividus

Strongylocentrotus droebachiensis

Spatangus purpureus

Echinocardium cordatum Echinocardium pennatifidium Echinocardium flavescens Meoma ventricosa

Brissopsis lyrifera

10) Asteroidea:

Anseropoda membranacea Solaster papposus

Echinaster sepositus Porania pulvillus Echinaster sanguinolentus Asterias rubens

grosser Seestern

Henricia leviuscula

Henricia sanguinolenta

Cribrella sanguinolenta

Luidia sarsi

Pentacerus hedemanni

\section{GAST}

Asterocheres violaceus Asterocheres violaceus Collocheres gracilicauda Asterocheres violaceus Asterocheres violaceus Asterocheres violaceus Asterocheres minutus Asterocheres violaceus Asterocheres violaceus Asterocheres violaceus Asterocheres violaceus Asterocheres violaceus Asterocheres violaceus Asterocheres minutus Asterocheres minutus Asterocheres violaceus Asterocheres violaceus Asterocheres violaceus Asterocheres violaceus

Asterocheres violaceus Asterocheres violaceus Micropontius ovioides Micropontius ovioides Scottomyzon gibberum Micropontius ovioides Micropontius ovioides Micropontius ovioides Micropontius ovioides Micropontius glaber Nanaspis pollens Micropontius ovioides

Asterocheres siphonatus Asterocheres violaceus Asterocheres lill jeborgi Asterocheres lilljeborgi Asterocheres violaceus Asterocheres lilljeborgi Asterocheres lilljeborgi Asterocheres violaceus Scottomyzon gibberum Scottomyzon gibberum Scottomyzon gibberum Scottomyzon gibberum Scottomyzon gibberum Scottomyzon gibberum Scottomyzon gibberum Asterocheres tenuicornis Asterocheres lillieborgi Asterocheres lilljeborgi Asterocheres lilljeborgi Asterocheres lilljeborgi Asterocheres lilljeborgi Asterocheres lilljeborgi Asterocheres lilljeborgi Asterocheres lilljeborgi Indomyzon qasimi Asterocheres sp.
ANZAHL

viele $\mathrm{Ex}$

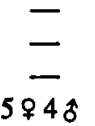

$5 \$ 4 \hat{\jmath}$

$34 \uparrow 18 \hat{8}$

viele Ex.
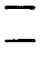

$-$

$-$

$=$

10 क 8

$40 \mathrm{Ex}$.

viele Ex. viele Ex. viele $q$ u 8 iele Ex. einige Ex. einige Ex. einige Ex. 136940 \& 20 के

128 einige Ex.

1 Ex. einige Ex.

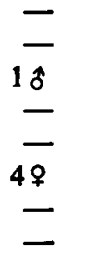

2 Ex. viele Ex.

-

19

T

$50 \mathrm{Ex}$

19 Ex.

3 Ex. 19

10 요 8 8 19
QUELLE

Bocquet, Stock \& Louise, 1963

Barel \& Kramers, 1970

Bocquet, 1952

Sars, 1915

Giesbrecht, 1897

Bocquet, Stock \& Louise, 1963

Bocquet, Stock \& Louise, 1963

Giesbrecht, 1897

Lang, 1949

Bresciani \& Lützen, 1962

Bocquet, 1952

Bocquet, Stock \& Louise, 1963

Barel, 1970

Claus, 1889

Giesbrecht, 1897

Claus, 1889

Giesbrecht, 1897

Bocquet, Stock \& Louise, 1963

Bocquet, 1952

Sars, 1915

Bresciani \& Lützen, 1962

Stock, 1960

Gooding, 1957

Gooding, 1957

Bresciani \& Lützen, 1962

Gooding, 1957

Gooding, 1957

Gooding, 1957

Stock, Humes \& Gooding, 1963

Stock, Humes \& Gooding, 1962

Gooding, 1957

Bocquet, 1952

Bresciani \& Lützen, 1962

Bocquet, 1952

Barel \& Kramers, 1970

Bresciani \& Lützen, 1962

Boeck, 1859

Sars, 1915

Lang, 1949

Th. \& A. Scott, 1895

Th. Scott, 1897

Bresciani \& Lützen, 1962

Stock, 1968

Bocquet, 1952

Barel \& Kramers, 1970

A. Scott, 1900

Brady, 1910

Wilson, 1944

Boeck, 1859

Lang, 1949

Bresciani \& Lützen, 1962

Bocquet, 1952

Barel \& Kramers, 1970

Hansen, 1923

Bresciani \& Lützen, 1962

Ummerkutty, 1966

Ummerkutty, 1966 


\section{WIRT}

11) Ophiuroidea:

Astroboa nuda

Astroboa albatrossi

Ophiothrix sp.

Ophiothrix quinquemaculata

Ophiothrix fragilis

Amphiura squamata

Amphodia urtica

Ophioderma longicauda

Ophioglypha sp.

Ophioglypha lacertosa

Ophiocoma nigra

Ophiocoma echinata

Ophiocantha vivipara

Ophiocantha disjuncta

Ophiopsila aranea

Amphiura sp.

Ophiactis sp.

12) Tunicata:

Corella parallelogramma

Phallusia virginea

Ascidien

kleine Ascidien
GAST

Collocherides astroboae

Collocherides astroboae

Asterocheres thompsoni

Collocheres gracilicaudo

Collocheres gracilicauda

Collocheres gracilicauda

Collocheres gracilicauda

Cancerilla tubulata

Cancerilla tubulata

Cancerilla tubulata

Cancerilla tubulata

Cancerilla tubulata

Cancerilla tubulata

Cancerilla tubulata

Cancerilla tubulata

Cancerilla tubulata

Cancerilla tubulata

Cancerilla tubulata

Cancerilla tubulata

Cancerilla tubulata

Cancerilla tubulata

Cancerilla tubulata

Cancerilla tubulata

Cancerilla tubulata

Cancerilla durbanensis

Cancerilla durbanensis

Cancerilla durbanensis

Cancerilla neozelanica

Cancerilla neozelanica

Cancerillopsis namaimensis

Cancerillopsis namaimensis

Collocheres breei

Asterocheres thompsoni

Asterocheres violaceus

Parartotrogus richardi

Cancerilla tubulata

Ophiopsyllus reductus

Cancerilla ampla

Cancerilla ampla

Cancerilla alata

Cancerilla alata

Cancerilla sp.

Cancerilla sp.

Parartotrogus richardi

Ophiopsyllopis indicus

Asterocheres siphonatus Asterocheres siphonatus Asterocheres siphonatus Asterocheres siphonatus Asterocheres ovalis Dyspontius striatus
ANZAHL

2396 웅

$1699 \AA$

一

viele Ex.

viele Ex.

viele Ex.

-

-

$-$

ב

二

$=$

\section{-}

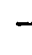

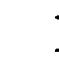

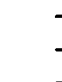

8 Ex.

-

-

1919

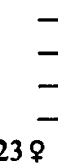

$23 \% 14$ oै
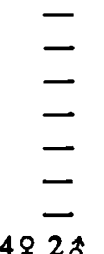

$4 \% 2$ \&

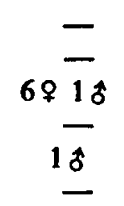

\section{QUELLE}

Stock, 1971

Stock, 1971

A. Scott, 1896

A. Scott, 1896

Stock, $1966 \mathrm{c}$

Stock, 1960

Stock, 1960; 1966c

Bresciani \& Lützen, 1962

Thompson, 1895

Giard, 1887

Giesbrecht, 1897

Stock, 1960

Bocquet, 1952

Gotto, 1963

Sars, 1918

Dalyell, 1851

Th. \& A. Scott, 1893

Thompson, 1895

Williams, 1954

Canu, 1891-92

Mercier, 1921

Cherbonnier, 1951

Cuénot, 1912

Claus, 1889

Zavodnik, 1958

Barnard, 1955

Gotto, 1963

Stephensen, 1933

Stephensen, 1927

Gotto, 1963

Gotto, 1963

Stock, 1966

A. Scott, 1896

A. Scott, 1896

Giesbrecht, 1897

Thompson, 1895

Stock, Humes \& Gooding, 1963

Gotto, 1963

Heegaard, 1951

Gotto, 1963

Heegaard, 1951

Heegaard, 1951

Changeux, 1957

Dalyell, 1851

Sebastian, 1968

Sars, 1915

Thorell, 1859

Lang, 1949

Aurivillius, 1882

Sewell, 1949

Canu, 1892 ad 1) Die Algen und Seegräser bilden, was ihre Gäste angeht, ein uneinheitliches Bild. Doch sind auch die meisten Angaben darïber nicht sehr genau. Allein die Angaben von Stock über Myzopontius australis auf Codium arabicum und von Lang über Dermatomyzon nigripes auf
Laminaria saccharina sind mit Zahlenangaben versehen. Doch sind diese sehr niedrig. Codium arabicum ist jedenfalls der einzige bekannte Wirt für Myzopontius australis.

ad 2) Die Kalkschwämme sind zum ersten Male im grösseren Ausmasse untersucht worden. Ihre 
Besprechung erfolgte schon weiter oben. Pericharax heteroraphis ist bisher der einzige und anscheinend auch der typische Wirt von Asterocheres proboscideus.

ad 3) Kieselschwämme:

a) Bei den Oscarella Arten ist sehr offensichtlich Asterocheres mucronipes vorherrschend. Wir haben hier sicher einen typischen Wirt vor uns.

b) Auf den Hadromerinen (Suberites, Cliona) finden wir Asterocheres echinicola und Asterocheres suberites, beide Vertreter mit sehr kurzem, birnförmigen Sipho.

c) Bei den Halichondria Arten fanden Lang und Klie viele Vertreter von Asterocheres parvus auf Halichondria sp. und Halichondria panicea, Stock 50 Exemplare von Asterocheres halichondriae auf Halichondria symbiotica. Typische Wirte liegen vor. Asterocheres echinicola dürfte auch ein vielgesehener Gast sein, vor allem auf Halichondria panicea, was die Funde von $\mathrm{Ha}$ mond und Stock beweisen.

d) Unter den Poecilosclerina gibt Stock Agelas orioides als typischen Wirt für Scottocheres laubieri an.

e) Bei der Gruppe der Haplosclerina ist Haliclona indistincta sicher der typische Wirt für Apodomyzon brevicorne und $A$. longicorne. Auf $P e$ trosia ficiformis wurden nur Cryptopontius Arten angetroffen.

ad 4) Unter den Angaben über die Hornschwämme finden wir zwei sicher typische Wirte: Ircinia muscarum für Psilomyzon pauciseta (Stock) und Dysidea tupha für Tuphacheres micropus (Stock).

ad 5) Anthozoa: sichere typische Wirte sind hier: Anemonia sulcata für Dinopontius acuticauda, Montipora sp. und Stylophora Arten für Asteropontius corallophilus, Dendrophyllia Arten für Cholomyzon palpiferum, Eunicella stricta für Acontiophorus bracatus.

ad 6) Mollusca: Boeck's Fund von Artotrogus orbicularis auf Doris sp. wurde noch nicht wiederholt. In gewisser Hinsicht bietet Lang mit seinem Fund von Art. orbicularis auf Nudibranchia eine Stütze. Chlamys opercularis ist bisher unter den Bivalven der einzige bekannte Wirt. ad 7) Auf den Crinoidea sind Collocheres Arten vorherrschend. Rosoll's Fund von Collocheres gracilicauda ist immer noch einzigartig. Stock fand einen typischen Wirt für Collocheres uncinatus in Heterometra savignyi.

ad 8) Holothuroidea: Allantogynus und Nanaspis Arten wurden hier gefunden. Isostichopus badionotus ist sicher ein typischer Wirt für $\mathrm{Na}$ naspis pollens, Stichopus regalis für N. mediterranea.

ad 9) Seeigel sind typische Wirte für Asterocheres violaceus; aber auch andere Arten mit sehr kurzem Sipho, wie Asterocheres echinicola und Asterocheres minutus, werden hier angetroffen. Offensichtlich eine Anpassung an den Wirt. Irreguläre Seeigel sind typisch für die zwei $M i$ cropontius Arten: Spatangus purpureus für Micropontius ovioides und Meoma ventricosa für Micropontius glaber. Calvocheres Arten sind ebenfalls nur hier zu finden.

ad 10) Asteroidea: Asterocheres lilljeborgi kommt häufig vor; Asterias rubens ist wohl sicher der typische Wirt für Scottomyzon gibberum.

ad 11) Ophiuroidea: Collocheres Arten sind hier in der Mehrheit. Stock gibt Ophiothrix fragilis als typischen Wirt für Collocheres gracilicauda an. Ophioderma longicauda ist der typische Wirt für Collocheres breei, Ophiocoma echinata für Ophiopsyllus reductus. Cancerilla tubulata und nahe Verwandte treffen wir häufig auf Amphiura squamata an. Astroboa nuda ist ein typischer Wirt für Collocherides astroboae.

ad 12) Tunicata dürften die Wirte für Asterocheres siphonatus sein, vor allem Corella parallelogramma.

\section{LITERATURVERZEICHNIS}

AURIVILlius, C. V. S., 1882. Bidrag till kännedorn om Krustaceer, som lefva hos Mollusker och Tunikater. Ofvers. K. Vet.-Akad. Forh., 39 (3) : 31-67, pls. V-VII \& 39 (8) : 41-117, pls. XIII-XVI.

Barel, C. D. N. \& P. G. N. Kramers, 1970. Notes on associates of echinoderms from Plymouth and the coast of Britany. Proc. kon. Nederl. Akad. Wetensch., Amsterdam, (C) 73 (2) : 159-170.

Barnard, K. H., 1955. South African Copepoda. Ann. S. Afr. Mus., 41: 223-312.
BocQuet, C., 1952. Copépodes sémi-parasites et parasites des Echinodermes de la région de Roscoff. Description de Lichomolgus asterinae n. sp. Bull. Soc. zool. France, 77 (5-6) : 495-504.

Bocquet, C., J. H. Stock \& F. Louise, 1963. Copépodes parasites d'Invertébrés des côtes de France, XV. Le problème systématique d'Asterocheres violacęus (Claus) et d'Asterocheres minutus (Claus). Proc. kon. Nederl. Akad. Wetensch., Amsterdam, (C) 66 (1) : $37-53$. 
BoECK, A., 1859. Beskrivelse over tvende nye parasitiske Krebsdyr, Artotrogus orbicularis og Asterocheres Liljeborgii. Forh. Vid. Selsk. Christiania, 1859 : 171182.

BraDY, G. S., 1880. A monograph of the free and semiparasitic Copepoda of the British Islands, $3: 1-83$, pls. 83-93. (Ray Soc., London).

-, 1910. Die marinen Copepoden der deutschen Südpolar-Expedition, 1901-1903, 1. Uber die Copepoden der Stämme Harpacticoida, Cyclopoida, Notodelphyoida und Caligoida. Deutsche Südpolar-Exped., 11 (Zool. 3) (5) : 497-593, pls. LII-LXIII.

Bresciani, J. \& J. LütZen, 1962. Parasitic copepods from the west coast of Sweden including some new or little known species. Vid. Medd. Dansk naturh. Foren., 124 : $367-408$.

BrIan, A., 1923a. Elenco di Copepodi marini bentonici proveniente da Rovigno e descrizione di una $n$. varietà di Parathalestris clausi Norm. Monitore zool. ital., 34 (7-8) : 126-135, pl. IV.

-, 1923b. Nota di Copepodi marini bentonici raccolti in Sardegna durante l'escursione organizzata dal Touring-Club Italiano nel maggio 1921. Atti Soc. Ital. Sci. nat., 62 : $176-178$.

-, 1928. Descrizione di specie nuove o poco conosciute di copepodi bentonici del mare Egeo. Boll. Mus. Zool. Anat. comp. R. Univ. Genova, 7 (18) : 1-37.

CanU, E., 1892. Copépodes du Boulonnais. Trav. Lab. Zool. Wimereux, $6: 1-354$, pls. I-XXX.

,- 1894 . Notes de biologie marine, faunistiques ou éthologiques, 1. Un Copépode Ascomyzontide sur une algue pélagique. Ann. Stat. aquic. Boulogne, 1: 100107 , pls. 6 et 7 .

-, 1898. Note sur les Copépodes et les Ostracodes marins des côtes de Normandie. Bull. soc. Amis Sci. natur. Rouen, (4) 33 : $389-422$, pl. III-X.

Changeux, J. P., 1957. Description de deux stades larvaires d'un Copépode Cancerillidae, parasite sur Ophiopsila aranea (Forbes). Vie Milieu, 8 (3) : 297311.

-, 1958. Quelques caractères biologiques d'un Copépode parasite d'Holothuries: Allantogynus delamarei, $n$. gen., n. sp. C.R. Acad. Sci. Paris, 247 : 961-964, 1 fig.

Cherbonnier, G., 1951. Echinodermes inventaire de la faune de Roscoff. Trav. Stat. biol. Roscoff, Suppl. 4 : $1-15$.

Claus, C., 1889. Uber neue oder wenig bekannte, halbparasitische Copepoden, insbesondere der Lichomolgiden und Ascomyzontiden-Gruppe. Arb. zool. Inst. Univ. Wien zool. Stat. Triest, 8 (3) : $327-370$, pls. I-VII.

CuÉnOT, L., 1912. Contribution à la faune du Bassin d'Arcachon, 5. Les Echinodermes. Bull. Stat. biol. Arcachon, 14 (1) : 17-116.

DALYELL, J., 1851. The powers of the creator displayed in the creation, 1: i-iv, $1-286$, pls. I-LXX. (J. van Voorst, London).

EISELT, J., 1961. Neubeschreibungen und Revision siphonostomer Cyclopoiden (Copepoda, Crust.) von der südlichen Hemisphäre nebst Bemerkungen über die Familie der Artotrogidae Brady, 1880. Anz. Oesterr. Akad. Wiss., (math.-naturw. K1.), 1961 (10) : 171174.
-, 1962. Neubeschreibungen und Revision siphonostomer Cyclopoiden (Copepoda, Crust.) von der südlichen Hemisphäre nebst Bemerkungen über die Familie der Artotrogidae Brady, 1880. Sitz. ber. Oesterr. Akad. Wiss., (math.-naturw. K1.) (1) 170 (7/10) : 315-366.

- , 1965. Revision und Neubeschreibungen weiterer siphonostomer Cyclopoiden (Copepoda, Crust.) aus der Antarktis. Sitz. ber. Oesterr. Akad. Wiss., (math.naturw. K1.) (1) 174 (3-4) : 151-169.

-, 1968. Revision von Acontiophorus antennatus Hansen 1923 und Materialien zur Variabilität von Acontiophorus armatus Brady 1880 (Cyclopoida Siph., Copepoda, Crust.). Sitz. ber. Osterr. Akad. Wiss., (math.naturw. K1.) (1) 177 (8) : 177-185.

Giard, A., 1887. Sur un Copépode, Cancerilla tubulata, Dalyell, parasite d'Amphiura squamata, Delle Chiaje, C.r. Acad. Sci., 104 : $1182-1192$.

Giesbrecht, W., 1895. The subfamilies, genera, and species of the copepod family Ascomyzontidae, Thorell, diagnosis, synonymy, and distribution. Ann. Mag. nat. Hist., (6) 16 : 173-186.

-, 1897. System der Ascomyzontiden, einer semi-parasitischen Copepoden-Familie. Zool. Anz., 20 : 9-14, 17-24.

-, 1899. Die Asterocheriden des Golfes von Neapel und der angrenzenden Meeres-Abschnitte. Fauna Flora Golfes Neapel, 25 : 1-217, pls. I-XI.

Gooding, R. U., 1957. On some Copepoda from Plymouth, mainly associated with invertebrates, including three new species. Journ. mar. biol. Ass. U.K., 36 : $195-221$.

Gotтo, R. V., 1963. Luminescent ophiuroids and associated copepods. Irish Natural. J., 14 (7) : 137-139.

GraefFe, E., 1902. Uebersicht der Fauna des Golfes von Triest ..., 5. Crustacea. Arb. zool. Inst. Univ. Wien, 13 (1) : 33-80.

GURNEY, R., 1927. Zoological results of the Cambridge Expedition to the Suez Canal, 1924, 33. Report on the Crustacea: Copepoda (littoral and semi-parasitic). Trans. zool. Soc. London, 22 (4) : 451-577.

HAMOND, R., 1968. Some marine copepods from Norfolk, Great Britain. Crustaceana, Suppl. 1: $38-48$.

HANSEN, H. J., 1902. Echinocheres globosus n. gen., n. sp., a copepod parasitic in spines of an echinothurid. Vidensk. Medd. Dansk naturh. Foren., (6) 4 : 437450, pl. XV.

-, 1923. Crustacea Copepoda, 2. Copepoda parasita and hemi-parasita. Ingolf Exped., 3 (7) : 1-92, pls. I-V.

HeEgaARD, P. E., 1951. Antartic parasitic copepods and an ascothoracid cirriped from brittle-stars. Vidensk. Medd. Dansk naturh. Foren., 113 : 171-190, 2 pls.

Humes, A. G. \& R. F. Cressey, 1959. A new family and genus of cyclopoid copepods parasitic on a holothurian. J. Parasitol., 45 : 209-216.

KLIE, W., 1933. Halbparasitische Copepoden aus der Kieler Bucht. Schr. Naturwiss. Ver. Schleswig-Holstein, 20 (1) : $12-17$.

KRIČ́AGIN, N., 1873. Otcet o faunisticeskikh izldovaniyakh proiz vedennykh ltom goda po poruceniyu kievskago obshchestva estestvoispytateley na, bepegakh Cernago Morya. Mém. Soc. Natural. Kiew, 3 (3) : 346-430, pls. $10-14$.

KRISHNASWAMY, S., 1959. Three semiparasitic copepods from the Madras coast. Rec. Ind. Mus., 54 (1/2) (1956) : $23-27$. 
LANG, K., 1949. On some Swedish marine semi-parasitic and parasitic copepods. Ark. Zool., 42A (22): 1-10.

Marcus, A., 1965. New Copepoda of the Black Sea. Trav. Mus. Hist. nat. „Gr. Antipa”, 5 : 91-96.

MERCIER, L., 1922. Copépode parasite d'une ophiure. Bull. Soc. Linn. Normandie, 4 : 17.

Murnane, J. P., 1967. A new family and genus of cyclopoid copepois associated with the sponge Cliona celata (Grant) in Massarhusetts, U.S.A. Crustaceana, 12 (3) : 225-232.

Nicholls, A. G., 1944. Littoral Copepoda from South Australia, (2): Calanoida, Cyclopoida, Notodelphyoida, Monstrilloida and Caligoida. Rec. S. Austr. Mus., 8 (1) : $1-62$.

Norman, A. M. \& T. Scotr, 1906. Crustacea of Devon and Cornwall : $\mathrm{i}-\mathrm{xv}, 1-232$, pls. I-XXIV. (W. Wesley and son, London).

RiedL, R., 1963. Fauna und Flora der Adria : 1-640, pls. I-VIII. (Paul Parey, Hamburg, Berlin).

Rosoll, A., 1889. Uber zwei neue an Echinodermen lebende parasitische Copepoden. Sitz. ber. math.naturw. Cl. Kais. Akad. Wiss. Wien, 97 (1) (4) : 188-202, pls. I-II.

SARS, G. O., 1915. An account of the Crustacea of Norway, 6. Copepoda Cyclopoida, pts. 7-10: 81-140, pls. XLIX-LXXX.

-, 1918. An account of the Crustacea of Norway, 6. Copepoda Cyclopoida, pts. 13-14: 173-225, pls. XCVII--CXVIII.

- , 1921. An account of the Crustacea of Norway, 7 Copepoda (Suppl.) : 1-114, pls. I-LXXVI.

SCoTT, A., 1896. Description of new and rare Copepoda. Rep. Lancashire Seafish. Lab. Univ. Coll. Liverpool, 1895 : $1-75$.

,- 1900 . Description and notes of some new and rare Copepoda from Liverpool Bay. Rep. Fauna Liverpool Bay, 5 : $59-83$.

ScotT, A. \& TH. ScotT, 1895. On some new and rare British Copepoda. Ann. Mag. nat. Hist., (6) 16 : 357358.

ScoTr, TH., 1897. Scottomyzon gibberum (Th. \& A. Scott) in the Cromarty Firth. Ann. Scot. nat. Hist., 23 : 195-196.

,- 1898 . Some additions to the invertebrate Fauna of Loch Fyne. Ann. Rep. Fish. Board Scotland, 16 : $261-282$.

ScotT, TH. \& A. ScotT, 1893. On some new or rare Crustacea from Scotland. Ann. Mag. nat. Hist., (6) 12 : 237-246, pls. XI-XIII.

Sebastian, M. J., 1968. On a new genus and species of cancerillid copepod, Ophiopsyllopsis indicus. Zool. Anz., 180 : $69-75$.

Sewell, R. B. S., 1949. The littoral and semiparasitic Cyclopoida, the Monstrilloida and Notodelphyoida. Sci. Rep. John Murray Exped. 1933/34, 9 (2) : 17199.

Stephensen, K., 1927. Papers from Dr. Th. Mortensen's Pacific Expedition 1914-16, XL. Crustacea from Auckland and Campbell Island. Vid. Medd. Dansk naturh. Foren., 83 : 289-390.

,- 1933 . Some new copepods, parasites on ophiurids and echinids. Vid. Medd. Dansk naturh. Foren, 93 : $197-$ 213.

,- 1933 . Some endoparasitic copepods found in echinids.
Vid. Medd. Dansk naturh. Foren., 98 : 223-228.

STOCK, J. H., 1960. Sur quelques Copépodes associés aux Invertébrés des côtes du Roussillon. Crustaceana, 1 (3) : 218-257.

-, 1965. Copépodes associés aux Invertébrés des côtes du Roussillon, 5. Cyclopoïdes siphonostomes spongicoles rares et nouveaux. Vie Milieu, $16(1-B)$ : 295324.

-, 1966a. Cyclopoida siphonostoma from Mauritius (Crustacea, Copepoda). Beaufortia, 13 (159) : 145194.

-, 1966b. Copepoda associated with invertebrates from the Gulf of Aqaba. Proc. kon. Ned. Akad. Wetensch., Amsterdam, (C) 69 (2) : 204-216.

-, 1966c. On Collocheres Canu, 1893, and Leptomyzon Sars, 1915, two synonymous genera of Copepoda. Beaufortia, 13 (163) : 221-239.

-, 1967. Copépodes associés aux Invertébrés des côtes du Roussillon, 7. Sur deux espèces jumelles de cyclopoïdes siphonostomes: Scottocheres elongatus (Th. \& A. Scott) et S. laubieri spec. nov. Vie Milieu, 18 (1-A) : 203-214.

-, 1968. Scottomyzon gibberum (Th. \& A. Scott, 1894), een parasitaire copepode van de zeester Asterias rubens L., in Nederland. Zeepaard, 28 (1) : 5-6.

,- 1968 . The Calvocheridae, a family of copepods inducing galls in sea-urchin spines. Bijdr. Dierk., 38 : $85-90$.

- 1970. Apodomyzon n. gen., a highly transformed siphonostome cyclopoid copepod, parasitic in the sponge Haliclona from Roscoff. Beaufortia, 18 (235) : $141-150$.

,- 1971. Collocherides astroboae n. gen., n. sp., a siphonostome cyclopoid copepod living in the stomach of basket stars. Bijdr. Dierk., 41 (1) : 19-22.

STrock, J. H. \& A. G. HumES, 1969. Cholomyzon palpiferum n. gen., n. sp., a siphonostome cyclopoid copepod parasitic in the coral Dendrophyllia from Madagascar. Crustaceana, 16 (1) : 57-64.

Stock, J. H., A. G. Humes \& R. U. Gooding, 1962. Copepoda associated with West Indian invertebrates, 1. The genus Nanaspis (Siph., Nanaspidae). Studies Fauna Curaçao, $13: 1-20$.

,$--\&-, 1963$. Copepoda associated with West Indian invertebrates, 2. Cancerillidae, Micropontiidae (Siphonostoma). Studies Fauna Curaçao, 15 : 1-23.

Stock, J. H. \& G. KLeeton, 1963a. Copépodes associés aux Invertébrés des côtes du Roussillon, 1. Cyclopoïdes associés aux Holothuries. Vie Milieu, 13 (4) : 681-701.

- \& -, 1963b. Copépodes associés aux Invertébrés des côtes du Roussillon, 3. Acontiophorus bracatus n. sp., un cyclopoïde siphonostome associé aux octocoralliaires. Vie Milieu, 14 : $551-560$.

Thompson, J. C., 1895. Recent additions to the Copepoda of Liverpool Bay. Trans. Liverpool biol. Soc., 9 : 95-103, pls. VI-VII.

THORELl, T., 1859. Bidri.g till kännedomen om Krustaceer, som lefva $\mathrm{i}$ arter af slägtet Ascidia L. K. Vet. Akad. Handl. Stockholm, 3: 1-84.

UMmerkUtTY, A. N. P., 1966. Studies on Indian copepods, 13. Brief notes on the asterocherid copepods obtained from the south-east coast of India with description of Indomyzon qasimi n. gen. n. sp. and a 
discussion on the family Asterocheridae. Crustaceana, 1 (1) : $17-32$, figs. $1-29$.

VìtovA, A., 1928. Compendio della flora e fauna del Mare Adriatico presso Rovigno con la distribuzione geografica delle specie bentoniche. Memoria R. Comitato thalassogr. Ital., 143 : 1-614, pls. I-LXVIII.

Williams, G., 1954. Fauna of Strangford Lough and neighbouring coasts. Proc. Roy. Irish Acad., 46 : 72.

Wilson, C. B., 1944. Parasitic copepods in the United
States National Museum. Prnc. U.S. nation. Mus., 94 : 529-582.

Yeatman, H. C., 1970. Copepods from Chesapeake Bay sponges including Asterccheres jeanyeatmanae n. sp. Trans. Americ. microsc. Soc., 89 (1) : 27-38.

ZavodNik, D., 1956. O kopepodu Cancerilla tubulata Dal., ektoparazitu na kacjerepu Amphipholis sqamata (D. Ch.). Biol. Vestnik, 7 : 81-83. 